\title{
MONODROMY OF CYCLIC COVERINGS OF THE PROJECTIVE LINE
}

\author{
T.N.VENKATARAMANA
}

\begin{abstract}
We show that the image of the pure braid group under the monodromy action on the homology of a cyclic covering of degree $d$ of the projective line is an arithmetic group provided the number of ramification points is sufficiently large compared to the degree $d$ and the ramification degrees are co-prime to $d$.
\end{abstract}

\section{INTRODUCTION}

A subgroup $\Gamma \subset G L_{N}(\mathbb{Z})$, is said to be an arithmetic group if $\Gamma$ has finite index in its integral Zariski closure $\mathcal{G}(\mathbb{Z})$ (i.e. suppose $\mathcal{G} \subset G L_{N}$ is the Zariski closure of $\Gamma$; then $\Gamma \subset \mathcal{G}(\mathbb{Z})$, which by definition, is $\mathcal{G} \cap G L_{N}(\mathbb{Z})$. We say that $\Gamma$ is arithmetic, if $\Gamma$ is a subgroup of finite index in $\mathcal{G}(\mathbb{Z})$ ). Otherwise, we say that $\Gamma$ is not arithmetic, or that $\Gamma$ is thin $[20$.

A natural class of subgroups of $S L_{N}(\mathbb{Z})$ arise as monodromy groups. Suppose $X \rightarrow S$ is a family of smooth projective varieties $\left(X_{s}\right)_{s \in S}$ parametrised by a base variety $S$. Then the fundamental group $\pi_{1}(S)$ acts on the integral cohomology $H^{*}\left(X_{s}\right)$ of a typical fibre $X_{s}$. The image of this action is the "monodromy group". Griffiths and Schmid 9] first raised the possibility that monodromy groups are arithmetic. However, there are several examples which show that the monodromy group is not always an arithmetic group. Notable among them are those of Deligne-Mostow [8] (see also [18] where the monodromy group is not even finitely presented). In the examples of [8, the monodromy group is a subgroup of infinite index in an arithmetic lattice in a product of unitary groups $U(r, s)$ (such that the group of the real points of the Zariski closure of the monodromy group contains the product of the special unitary groups $S U(p, q)$ and ) such that one of the factors of the product is $U(n-1,1)$. The projection of the monodromy to this factor sometimes gives a lattice in $U(n-1,1)$ which can be shown to

1991 Mathematics Subject Classification. primary: 22E40. Secondary: 20F36 T.N.Venkataramana, School of Mathematics, Tata Institute of Fundamental Research, Homi Bhabha Road, Colaba, Mumbai 40005, INDIA. 
be a non-arithmetic lattice in $U(n-1,1)$.

The examples of [8] arise as the monodromy of certain families of cyclic coverings of a fixed order $d$ of the projective line $\mathbb{P}^{1}(\mathbb{C})$, where the family is prescribed by choosing $n+1$ distinct branch points in the affine line $\mathbb{C}$, with fixed ramification. To be precise, let $n \geq 1$ and $d \geq 2$ be integers. Fix integers $k_{1}, k_{2}, \cdots, k_{n+1}$ with $1 \leq k_{i} \leq d-1$, and such that the g.c.d. of $k_{1}, k_{2}, \cdots, k_{n+1}$ and $d$ is 1 . Given $n+1$ distinct points $a_{1}, a_{2}, \cdots, a_{n+1}$ in the complex plane, put $a=\left(a_{1}, \cdots, a_{n+1}\right) \in \mathbb{C}^{n+1}$. Consider the curve $X_{a, k}$ given by the pair $(x, y)$ satisfying the equation

$$
y^{d}=\left(x-a_{1}\right)^{k_{1}}\left(x-a_{2}\right)^{k_{2}} \cdots\left(x-a_{n+1}\right)^{k_{n+1}}
$$

with $y \neq 0$ and $x \neq a_{1}, a_{2}, \cdots, a_{n+1}$.

Let $\mathcal{C}$ be the space of points in $\mathbb{C}^{n+1}$ all of whose coordinates are distinct; as the point $a \in \mathcal{C}$ varies, we get a family

$$
\mathcal{F}=\left\{(y, x, a) \in \mathbb{C}^{*} \times \mathbb{C} \times \mathcal{C}: y^{d}=\prod_{i=1}^{n+1}\left(x-a_{i}\right)^{k_{i}}\right\},
$$

and the fibration $\mathcal{F} \rightarrow \mathcal{C}$ given by the projection map $(y, x, a) \mapsto a$. The fibre over a point $a \in \mathcal{C}$ is the affine curve given in equation (1). The curve $X_{a, k}$ is a compact Riemann surface $X_{a, k}^{*}$ minus a finite set of punctures. We may consider, analogously, the family $\mathcal{F}^{*}$ of compact Riemann surfaces $X_{a, k}^{*}$ fibering over $\mathcal{C}$.

The fundamental group of the space $\mathcal{C}$ is well known to be the pure braid group $P_{n+1}$ on $n+1$ strands (see subsection 7.1); thus the fibration $\mathcal{F}^{*} \rightarrow \mathcal{C}$ yields a monodromy representation

$$
\rho_{M}^{*}(k, d): P_{n+1} \rightarrow G L\left(H_{1}\left(X_{a, k}^{*}, \mathbb{Z}\right)\right),
$$

of $P_{n+1}$ on the integral homology of the fibre $X_{a, k}^{*}$. If $N$ is the rank of the abelian group $H_{1}\left(X_{a, k}^{*}, \mathbb{Z}\right)$, then the image $\Gamma$ of $P_{n+1}$ is a subgroup of $G L_{N}(\mathbb{Z})$. It can be shown that the group $\mathcal{G}(\mathbb{R})$ of real points of the Zariski closure of $\Gamma$ is contained in a product of unitary groups $U(p, q)$ such that $\mathcal{G}(\mathbb{R})$ contains the product of the special unitary groups $S U(p, q)$. The group $G=\mathbb{Z} / d \mathbb{Z}$ acts on the equation

$$
y^{d}=\prod_{i=1}^{n+1}\left(x-a_{i}\right)^{k_{i}}
$$

by the map $g(x, y) \mapsto g y$ for $g \in G$ where $G$ is viewed as $d$-th roots of unity in $\mathbb{C}$. We may decompose the homology $H_{1}$ of $X_{a, k}^{*}$ into $G$ eigenspaces with respect to this action. Fix a primitive $d$-th root of 
unity, say $\omega=e^{2 \pi i / d}$. Fix a generator $T$ of $G$. If $1 \leq f \leq d$ is an integer, fix the part of the homology $H_{1}\left(X_{a, k}^{*}, \mathbb{C}\right)$ on which the generator $T \in G$ acts by the scalar $\omega^{f}$. The group of real points of the Zariski closure of $\Gamma$ acting on this part will again be contained in a unitary group of the form $U\left(p_{f}, q_{f}\right)$ and will (in general) contain the special unitary group $S U\left(p_{f}, q_{f}\right)$.

We now describe briefly, the results of [8]. Suppose $f$ is an integer with $1 \leq f \leq d-1$, and coprime to $d$. Given $x \in \mathbb{R}$, denote by $\{x\}$ its fractional part. Put $\mu_{i}=\left\{\frac{k_{i} f}{d}\right\}$ for $1 \leq i \leq n+1$. Write $\mu_{\infty}=2-\sum \mu_{i}$. We impose the following conditions on the $\mu_{i}$. [1] $\mu_{i}+\mu_{j}<1$ for all $i, j$ including $i=\infty$ [2] $0<\mu_{\infty}$ [3] $\frac{1}{1-\mu_{i}-\mu_{j}}$ is an integer if $k_{i} \neq k_{j}$ [4] if $k_{i}=k_{j}$ then $\frac{1}{1-2 \mu_{i}}$ is a half integer.

Then it is shown in [8] that the factor of the group of real points of the Zariski closure of the monodromy $\Gamma$ in $G L\left(H_{1}\left(X_{a, k}^{*}, \mathbb{Z}\right)\right)=G L_{N}(\mathbb{Z}) \subset$ $G L_{N}(\mathbb{C})$ corresponding to $f$ (as in the preceding paragraph) contains the special unitary group $S U(n-1,1)$ and is contained in $U(n-1,1)$. Moreover, the projection of $\Gamma$ to this factor gives a lattice in $U(n-1,1)$ (if the $\mu_{i}$ satisfy some further conditions, then the lattice in $U(n-1,1)$ is an arithmetic lattice).

For example, consider the family for varying $b_{1}, b_{2}, b_{3}, b_{4} \in \mathbb{C}$, all distinct, of the curves corresponding to the equation

$$
y^{18}=\left(x-b_{1}\right)\left(x-b_{2}\right)\left(x-b_{3}\right)\left(x-b_{4}\right) .
$$

In this case, $n=3$. By the criteria of [8], the monodromy is nonarithmetic. In the notation of the preceding paragraph, we take $f=7$ and $d=18$; then $\mu_{i}=7 / 18$ and $\mu_{\infty}=8 / 18$. Hence $\frac{1}{1-\mu_{i}-\mu_{j}}$ is a half integer -namely $9 / 2$ - if $i, j \leq 4$ (and hence $\mu_{i}=\mu_{j}$ ); and $\frac{1}{1-\mu_{i}-\mu_{j}}$ is an integer- namely 6 - if $i \leq 4$ and $j=\infty$. By the half integrality $(\Sigma-I N T)$ criterion of Mostow (in [17], see page 104, with $N=5$ and $\mu_{i}=7 / 18$, and $\left.\mu_{\infty}=8 / 18\right)$ it follows that the projection of $\Gamma$ to the factor corresponding to $f=7$ is a discrete subgroup of $U(2,1)$ and is, in fact, a lattice in $U(2,1)$. One can easily check, from the list given there, that the monodromy is non-arithmetic i.e. has infinite index in its integral Zariski closure.

Let us now return to the general situation of equation (1). The condition of [8] that $0<\mu_{\infty}=2-\sum\left\{\frac{k_{i} f}{d}\right\}$ implies that $n+1 \leq \sum k_{i} \leq$ $2 d$ and hence that $n \leq 2 d-1$. We would like to investigate what 
happens when $n \geq 2 d$. The following theorem says that if $n \geq 2 d$ then for most $k_{i}$ 's, the monodromy is arithmetic. Precisely, we prove

Theorem 1. Suppose $d \geq 2$ and $n \geq 1$ are integers, $k_{1}, \cdots, k_{n+1}$ are integers with $1 \leq k_{i} \leq d-1$ with g.c.d $\left(k_{i}, d\right)=1$ for each $i$. Suppose that

$$
n \geq 2 d \text {. }
$$

Then the image $\Gamma=\rho_{M}^{*}(k, d)\left(P_{n+1}\right)$ of the monodromy representation $\rho_{M}^{*}(k, d)$ of $P_{n+1}$ is an arithmetic group.

Moreover, the monodromy group is (up to finite index) a product of irreducible lattices each of which has $\mathbb{Q}$-rank at least two.

In [25], the case when all the integers $k_{i}$ are 1 was considered (then g.c.d $\left(k_{i}, d\right)=1$ for all $\left.i\right)$. Consider the compactification $X_{a}^{*}$ of the affine curve

$$
y^{d}=\left(x-a_{1}\right)\left(x-a_{2}\right) \cdots\left(x-a_{n+1}\right),
$$

with $y \neq 0$ and $x \neq a_{1}, \cdots, a_{n+1}$. There is now the monodromy action of the pure braid group $P_{n+1}$ (even of the full braid group $B_{n+1}$ ) on $H_{1}\left(X_{a}^{*}, \mathbb{Z}\right)$. The following result is proved in [25].

Theorem 2. If $d \geq 3$ and $n \geq 2 d$, then the image $\Gamma$ of the monodromy representation $\rho(d): B_{n+1} \rightarrow G L\left(H_{1}\left(X_{a}^{*}, \mathbb{Z}\right)\right)=G L_{N}(\mathbb{Z})$ is an arithmetic group.

Moreover, the monodromy is a finite index subgroup of a product of irreducible lattices, each of which is a non-co-compact arithmetic group and has $\mathbb{Q}$-rank at least two.

Remark 1. If $n+1 \leq d$ then the group of integral points of the Zariski closure of the monodromy is (up to finite index) a product of irreducible arithmetic lattices, some of which form co-compact lattices of their real Zariski closures.

A result of A'Campo [3] says that Theorem 2 holds when $d=2$ as well.

If we replace the pure braid group by the mapping class group $\Gamma_{g}$ of the fundamental group of a compact Riemann surface of genus $g \geq 2$, and consider analogously, the action of $\Gamma_{g}$ on the family of cyclic coverings of a fixed degree of the family of genus $g$ Riemann surfaces, then the arithmeticity of the image of this action (monodromy) is proved in [14] (at the time the present article was written, the author was not 
aware of the paper [14]; the method of proof is similar and uses the presence of unipotent elements in the monodromy group. But, in the present article, more work is needed to generate unipotent elementsunder the assumption that $n \geq 2 d$ ).

A special case of Theorem 1 is the following

Corollary 1. Supposed is a prime, $k_{1}, \cdots, k_{n+1}$ integers with $1 \leq k_{i} \leq$ $d-1$ and $n \geq 2 d$. Then the monodromy group $\Gamma$, namely the image of $P_{n+1}$ under the representation $\rho(k, d): P_{n+1} \rightarrow G L\left(H_{1}\left(X_{a, k}^{*}, \mathbb{Z}\right)\right)$ is an arithmetic group.

Remark 2. If $d$ is not assumed to be prime, then the analogue of Corollary 1 is false in general, even when $n$ is large. As an example, consider $d=2 \times 18$ and let $n$ be arbitrary. Suppose $a_{1}, a_{2}, \cdots, a_{n}, b_{1}, b_{2}, b_{3}, b_{4}$ are distinct complex numbers. Consider the two equations

$$
\begin{gathered}
C_{d}(a, b): y^{2 \times 18}=\left(\prod_{i=1}^{n}\left(x-a_{i}\right)\right)^{18}\left(x-b_{1}\right) \cdots\left(x-b_{4}\right) \text { and } \\
C_{18}(b): w^{18}=\left(x-b_{1}\right) \cdots\left(x-b_{4}\right) .
\end{gathered}
$$

There is a map $C_{2 \times 18}(a, b) \rightarrow C_{18}(b)$ given by $(x, y) \mapsto(x, w)$ with

$$
w=\frac{y^{2}}{\left(x-a_{1}\right) \cdots\left(x-a_{n}\right)} .
$$

The monodromy of the family $C_{2 \times 18}(a, b)$ (as $a$ and $b$ vary) on the first homology of the curves $C_{2 \times 18}(a, b)$ maps onto the corresponding monodromy of the family of the curves $C_{18}(b)$ (as $b$ varies). The latter is not arithmetic, by the example discussed earlier. Therefore, the monodromy of the family $C_{2 \times 18}(a, b)$ is also non-arithmetic.

1.1. Description of the Proof. The proof is very similar to the proof of Theorem 2 given in [25]. In [25] the proof was by showing that the monodromy was related to the Burau representation. The properties of the Burau representation (especially those at roots of unity) were used in the course of the proof.

Analogously, in the present paper, Theorem 1 is deduced from the arithmeticity of the images of certain representations (the reduced Gassner representation specialised at roots of unity defined in subsection 3.4) of the pure braid group $P_{n+1}$. We also have to establish, somewhat precisely, the exact relationship of the monodromy in Theorem 1 with the Gassner representation. This is much more complicated than in the Burau case. The monodromy representation of Theorem [1] is related to the reduced Gassner representation of Theorem [16] as 
follows (see [11] for related results).

One can define the reduced Gassner representation $g_{n}(k, d)$ at $d$ th roots of unity where $k$ is the $n+1$-tuple $\left(k_{1}, \cdots, k_{n+1}\right)$. The image of $g_{n}(k, d)$ takes the pure braid group $P_{n+1}$ into $G L_{n}\left(E_{d}\right)$ where $E_{d}=\mathbb{Q}\left(\omega_{d}\right)$ is the $d$-th cyclotomic extension. We will see in section 4 that the Gassner representation $g_{n}(k, d)$ is irreducible if $\sum k_{i}$ is not divisible by $d$; if $\sum_{i} k_{i}$ is divisible by $d$, then $g_{n}(k, d)$ contains the one dimensional trivial representation $E_{d} v$ and the quotient, denoted $\bar{g}_{n}(k, d)$, is irreducible. By an abuse of notation, we write $\bar{g}_{n}(k, d)$ for the representation $g_{n}(k, d)$ even when $\sum k_{i}$ is not divisible by $d$.

If $X_{a, k}$ is the open curve, then we have the monodromy action, denoted $\rho_{M}(k, d)$ on $H_{1}\left(X_{a, k}, \mathbb{Q}\right)$ (and the action $\rho_{M}^{*}\left(X_{a, k}^{*}, \mathbb{Q}\right)$ on the homology of the projective curve $X_{a, k}^{*}$ ). On the homology of $X_{a, k}$ the cyclic group $\mathbb{Z} / d \mathbb{Z}$ operates. Given a module $V$ of the $\mathbb{Q}$-group algebra $\mathbb{Q}[\mathbb{Z} / d \mathbb{Z}]$, denote by $V^{n i}$ the quotient of $V$ modulo the space of invariant vectors in $V$ under the action of $\mathbb{Z} / d \mathbb{Z}$. Take $V=H_{1}\left(X_{a, k}, \mathbb{Q}\right)$. We call $V^{n i}$ the "non-invariant" part of $V$ and denote by $\rho_{M}(k, d)^{n i}$ the representation of $P_{n+1}$ on $V^{n i}$. In section 7 , we will prove

Proposition 3. Suppose that the numbers $k_{i}$ are all co-prime to $d$. Denote by $\rho_{M}(k, d)$ the representation of the pure braid group $P_{n+1}$ on the homology of the open curve $H_{1}\left(X_{a, k}, \mathbb{Q}\right)$. Then the non-invariant part of $H_{1}\left(X_{a, k}, \mathbb{Q}\right)$ is the direct sum

$$
\rho_{M}(k, d)^{n i}=\bigoplus_{e \mid d} g_{n}(k, e) .
$$

The representation $\rho_{M}^{*}(k, d)$ of $P_{n+1}$ on the homology $H_{1}\left(X_{a, k}^{*}, \mathbb{Q}\right)$ of the compact Riemann surface $X_{a, k}^{*}$ is the direct sum

$$
\rho_{M}^{*}(k, d)=\bigoplus_{e \mid d} \bar{g}_{n}(k, e) .
$$

The sum is over all divisors $e \geq 2$ of the integer $d$.

Theorem 1 follows from Proposition 3 and Theorem 16.

The main section of the paper is Section 4, In section 4, we show that the image of the pure braid group $P_{n+1}$ at a primitive $d$-th root of unity contains many unipotent elements. More precisely, the proof of Theorem 16 is by showing that for $n \geq 2 d$ the image $\Gamma_{n}(q)$ contains an arithmetic subgroup of the unipotent radical of a parabolic $\mathbb{Q}$ - subgroup. By using results of Bass-Milnor-Serre and Tits ([4], 22]), and 
their extensions to other groups ([19], [23], [24]) on unipotent generators for noncocompact arithmetic groups of $\mathbb{R}$-rank at least two, one can then show that such groups are arithmetic if $n \geq 2 d$.

In the Burau case, this was proved in [25]. It was possible to obtain unipotent elements in the Burau case when $n+1$ was divisible by $d$, since in that case, the Burau representation at $d$-th roots of unity is degenerate. The unitary group $U(h)$ of the relevant hermitian form is not reductive and we can get, in the image of the Burau representation, elements which lie in the unipotent radical of $U(h)$.

An analogous result in the Gassner case is proved in the present paper. We exploit the fact that (if $n \geq 2 d$ ) then a subrepresentation of the restriction of the Gassner representation at roots of unity, to a suitable smaller pure braid group, becomes degenerate. One can then generate unipotent elements. The existence of such a suitable smaller pure braid group is ensured by a pigeon-hole argument if $n \geq 2 d$. This is worked out in Section 4 .

In section 6 we relate the Gassner representation to the pure braid action on certain finite index subgroups of the free group on $n+1$ generators. This relation is obtained by using a Theorem of Artin on the action of the (pure) braid group on the free group on $n+1$ generators. We then relate this action to the monodromy in section 7 . 


\section{Contents}

1. Introduction

1.1. Description of the Proof

2. Algebraic Groups

2.1. An inductive step for integral unitary groups 9

2.2. Groups Generated by Complex Reflections 10

2.3. Some results on algebraic groups 13

2.4. Products 15

3. Action of the Braid Group on a Free Group 15

3.1. The pure braid group 16

3.2. Artin's Theorem 16

3.3. Action on Certain Subgroups and Sub-quotients 17

3.4. The Gassner Representation $\quad 17$

3.5. An invariant element in the Gassner representation 23

3.6. A supplement to the space of invariants 23

4. Properties of the The Gassner Representation 24

4.1. A (skew) hermitian form preserved by the pure braid group 24

4.2. Irreducibility 27

5. Proof of Theorem 16 30

5.1. The reduced Gassner representation at roots of unity 30

5.2. Gassner representations with degenerate Hermitian forms 31

5.3. Proof of Theorem 16

6. Homology of Cyclic Coverings 38

6.1. Image of homology 38

6.2. Image of the Gassner Module 40

7. Connection with Monodromy 44

7.1. Some Cyclic Coverings of $\mathbb{P}^{1}$

7.2. The Compactification of $X_{a, k} \quad 45$

References 46

\section{Algebraic Groups}

The following theorem is an extension to all simple groups, and all opposing parabolic subgroups, of a result of Bass-Milnor-Serre and of Tits (the theorem of Bass-Milnor-Serre and Tits was proved for $S L_{n}$ $(n \geq 3)$ and $\left.S p_{2 g}(g \geq 2)\right)$, and where the parabolic subgroup was a minimal parabolic subgroup. We refer to section 2 of [25] for a detailed description and definitions of the terms involved. 
Theorem 4. Suppose $G$ is an absolutely almost simple linear algebraic group defined over a number field $K$, such that $K$-rank of $G$ is $\geq 1$ and $G\left(O_{K}\right)$ has higher real rank, i.e.

$$
\infty-\operatorname{rank}(G) \stackrel{\text { def }}{=} \sum_{v \mid \infty} K_{v}-\operatorname{rank}(G) \geq 2 .
$$

Suppose $P$ is a parabolic $K$-subgroup of $G$ with unipotent radical $U$ and let $P^{-}$be a parabolic $K$-subgroup defined over $K$ and opposed to $P$ with unipotent radical $U^{-}$. Let $\Gamma \subset G\left(O_{K}\right)$ be a subgroup which intersects $U\left(O_{K}\right)$ in a finite index subgroup ( and similarly with $U^{-}\left(O_{K}\right)$ ). Then $\Gamma$ has finite index in $G\left(O_{K}\right)$.

2.1. An inductive step for integral unitary groups. In this subsection, we prove a result which will be used in the inductive proof of Theorem 1. The result says that a subgroup of the integral unitary group has finite index if it contains finite index subgroups of smaller integral unitary groups. In the following, we will assume that $E$ is a totally imaginary quadratic extension of a totally real number field $K$. Let $x \mapsto \bar{x}$ denote the action of the non-trivial element of the Galois group of the quadratic extension $E / K$. Assume that $V$ is a finite dimensional $E$-vector space and that $h: V \times V \rightarrow E$ a $K$ bilinear form such that $h(\lambda v, \mu w)=\lambda \bar{\mu} h(v, w)$ for all $\lambda, \mu \in E$ and all $v, w \in V$. Assume that $h(w, v)=\bar{h}(v, w)$ for all $v, w \in V$. Then the unitary group $U(h)$ (resp. the special unitary group $S U(h)$ ) of elements of $G L(V)$ (resp. $S L(V)$ ) which preserve $h$ is naturally an algebraic group (resp. an almost simple algebraic group) over the totally real number field $K$. Under suitable conditions, we will be able to apply Theorem 4 to $S U(h)$.

Moreover, if $K_{v} \simeq \mathbb{R}$ is an archimedean completion of the (totally real) number field $K$, then the base change of $U(h)$ to $K_{v}$ is the usual unitary group of the Hermtitian form over $\mathbb{R}$. In particular, the special unitary group $S U(h)\left(K_{v}\right)$ is a co-compact subgroup of $U(h)\left(K_{v}\right)$. As a consequence, the group of integral points $U(h)\left(O_{K}\right)$ and $S U(h)\left(O_{K}\right)$ are commensurable. Therefore, arithmetic subgroups of $U(h)$ or of $S U(h)$, are the same up to commensurability.

We note that in our applications, the goups involved will be unitary groups of skew hermitian forms; but these are naturally isomorphic to unitary groups of hermitian forms, by changing the skew form by a multiple of an imaginary element. For this reason, we do not stress the nature of the form, whether it is hermitian or skew hermitian. 
Notation. With the preceding assumptions, Let $V=(V, h)$ be a nondegenerate hermitian space over $E$ such that $E-\operatorname{rank}(V, h) \geq 2$; since the special unitary group is a $K$-group, this hypothesis is equivalent to $K-\operatorname{rank}(S U(h)) \geq 2$. Let $W, \quad W^{\prime}$ be codimension one subspaces on which $h$ is again non-degenerate. Suppose that $\Gamma \subset H_{V}\left(O_{K}\right)$ is a subgroup such that its intersection with $U_{W}\left(O_{K}\right)\left(\operatorname{resp} U_{W^{\prime}}\left(O_{K}\right)\right)$ has finite index in $U_{W}\left(O_{K}\right)$ (resp. in $U_{W^{\prime}}\left(O_{K}\right)$ ).

Lemma 5. With the preceding notation, suppose that there exists a non-degenerate subspace $W^{\prime \prime}$ of the intersection $W \cap W^{\prime}$ which contains a nonzero isotropic vector $v$. Then the group $\Gamma$ has finite index in $U_{V}\left(O_{K}\right)$.

Proof. By the non-degeneracy of $h$ on $W^{\prime \prime} \subset W \cap W^{\prime}$ the space $W^{\prime \prime}$ contains a vector $v^{*}$, also isotropic, such that $h\left(v, v^{*}\right)=1$. Write the orthogonal decomposition $V=\left(E v+E v^{*}\right) \oplus X$. Then $W=$ $\left(E v+E v^{*}\right) \oplus X \cap W$ and similarly for $W^{\prime}$.

Consider the filtration

$$
0 \subset E v \subset E \oplus X \subset E v \oplus X \oplus E v^{*}=V .
$$

Denote the corresponding Heisenberg group (the unipotent subgroup of $U(V)$ which preserves the flag and acts by identity on successive quotients), by $H(V)$ and its integral points by $H(V)\left(O_{K}\right)=H_{V}\left(O_{K}\right)$. The group $P \subset U(V)$ which preserves the above partial flag is a parabolic subgroup and $H(V)$ is its unipotent radical. Define similarly, the smaller Heisenberg groups $H(W)$ and $H\left(W^{\prime}\right)$ and their integral points $H(W)\left(O_{K}\right)$ and $H\left(W^{\prime}\right)\left(O_{K}\right)$.

By assumption, $H(W) \cap \Gamma$ has finite index in $H(W)\left(O_{K}\right)$; similarly for $H\left(W^{\prime}\right)$. The two integral Heisenberg groups generate $H(W)\left(O_{K}\right)$ up to finite index, since two distinct vector subspaces of codimension one, span the whole space. We thus find that $\Gamma$ contains a subgroup of finite index in the integral unipotent radical of a parabolic $K$ subgroup.

Similarly, we find a finite index subgroup of an opposite integral unipotent radical in the group $\Gamma$. Therefore, by Theorem 4 applied to $S U(h), \Gamma \cap S U(h)$ is arithmetic. Since $U(h)\left(O_{K}\right)$ and $S U(h)\left(O_{K}\right)$ are commensurable, it follows that $\Gamma$ is an arithmetic subgroup of $U(h)\left(O_{K}\right)$.

2.2. Groups Generated by Complex Reflections. The results in this subsection deal with irreducibility of the action of groups generated by complex reflections on a complex vector space (sometimes ones 
equipped with a hermitian form). They are essentially well known, but we need a version involving additive subgroups of vector groups stable under complex reflections and therefore we record them here.

Let $V$ be an $n$-dimensional vector space over a field $K$. We say that an element $T \in G L(V)$ is a generalised reflection if the endomorphism $T-1$ has one dimensional image. Suppose that $T_{1}, \cdots, T_{n}$ are generalised reflections such that $\left(T_{i}-1\right)(V)=K \varepsilon_{i}$ and $\left\{\varepsilon_{i}: 1 \leq i \leq n\right\}$ form a basis of $V$. Assume that for each $i \leq n-1,\left(T_{i}-1\right)\left(\varepsilon_{i+1}\right)=b_{i} \varepsilon_{i}$ with $b_{i} \neq 0$. Assume also that if $i \geq 2$ then $\left(T_{i}-1\right)\left(\varepsilon_{i-1}\right)=a_{i} \varepsilon_{i}$ with $a_{i} \neq 0$.

Lemma 6. [1] Let $V$ and $T_{i}$ be as in the preceding and $\Delta$ the group generated by the transformations $\left\{T_{i} ; 1 \leq i \leq n\right\}$. Denote by $V^{\Delta}$ the space of vectors in $V$ invariant under $\Gamma$. Then the quotient $V / V^{\Delta}$ is an irreducible representation of $\Delta$.

[2] If in addition, we assume that $T_{i}\left(\varepsilon_{j}\right)=\varepsilon_{j}$ for all $i, j$ with $|i-j| \geq$ 2 , then the space $V^{\Delta}$ of invariant vectors has dimension at most one.

Proof. Suppose that $W \neq V$ is a $\Delta$ invariant subspace. If $\varepsilon_{j} \in W$ for some $j$, by the $T_{j-1}$ invariance of $W$, the vector $\left(T_{j-1}-1\right)\left(\varepsilon_{j}\right)$ lies in $W$. By assumption, $\left(T_{j-1}-1\right) \varepsilon_{j}$ is a non-zero multiple of $\varepsilon_{j-1}$; therefore, $W$ contains $\varepsilon_{j-1}$. Similarly, $\left(T_{j+1}-1\right) \varepsilon_{j} \in W$ and is a non-zero multiple of $\varepsilon_{j+1}$ if $j \leq n-1$. Therefore, if $\varepsilon_{j}$ lies in $W$ for some $j$, then $\varepsilon_{1}, \cdots, \varepsilon_{n}$ lie in $W$ and hence $W=V$, a contradiction.

Consequently, $W$ does not contain $\varepsilon_{j}$ for any $j$. Consider the image $\left(T_{i}-1\right) W \subset W$. If the image is non-zero, then it consists of all multiples of $\varepsilon_{i}$ and this is impossible by the preceding paragraph. Therefore $\left(T_{i}-1\right) W=0$, which means that $T_{i}$ is identity on $W$ for every $i$. In other words, $W$ is contained in $V^{\Delta}$. This proves part [1] of the lemma.

We will now prove part [2], assuming (as in part [2]) that $T_{i}\left(\varepsilon_{j}\right)=\varepsilon_{j}$ if $|i-j| \geq 2$. Suppose that $v \in V^{\Delta}$ is of the form $v=x_{2} \varepsilon_{2}+\cdots+x_{n} \varepsilon_{n}$ (i.e. the coefficient $x_{1}$ of $\varepsilon_{1}$ is zero). Applying $\left(T_{1}-1\right)$ to $v$ we get $0=\left(T_{1}-1\right) v=x_{2} b_{1} \varepsilon_{1}$ whence $x_{2}=0$. Now applying $T_{2}-1$ to $v$, we get $0=\left(T_{2}-1\right) v=x_{3} b_{2} \varepsilon_{2}$. Therefore $x_{3}=0, \cdots$. An easy induction now establishes that all the $x_{i}$ are zero. Hence the linear map $V^{\Delta} \rightarrow K$ given by $v=\sum_{i=1}^{n} x_{i} \varepsilon_{i} \mapsto x_{1}$ (the first coordinate function) is injective. Therefore the second part of the lemma follows. 
We now prove a version of Lemma 6 for additive subgroups of a vector group stable under the $T_{i}$. Let $A$ be an integral domain and $\Omega$ a field of characteristic zero containing $A$; suppose there is an involution of the field $\Omega$ (field automorphism of order two) which stabilises $A$ and $V$ a finite dimensional $\Omega$ vector space of dimension $n$ with a non-degenerate hermitian form $h$ with respect to this involution. Suppose that $\left\{T_{i} \in G L(V): 1 \leq i \leq n\right\}$ preserve this hermitian form such that the space of vectors fixed under $T_{i}$ is of codimension one; then the image of $T_{i}-1$ is spanned by the unique (up to scalar multiples) eigenvector for $T_{i}$ with eigenvalue not 1 , denote it $\varepsilon_{i}$.

We will assume that the $\left\{\varepsilon_{i}: 1 \leq i \leq n\right\}$ form a basis of $V$, and that for each $i$,

$$
\begin{aligned}
& T_{i}\left(\varepsilon_{i+1}\right)=a_{i} \varepsilon_{i+1}+b_{i} \varepsilon_{i} \text { with } b_{i} \neq 0, \\
& T_{i}\left(\varepsilon_{i-1}\right)=c_{i} \varepsilon_{i}+d_{i} \varepsilon_{i-1} \text { with } c_{i} \neq 0 .
\end{aligned}
$$

Under these assumptions we have the

Lemma 7. Let $\Gamma \subset U(h)(A) \subset U(V)$ be a subgroup generated by these complex reflections $T_{i}$. Let $W$ be an additive subgroup of the vector group $V$, such that $W$ is stable under the operators $T_{i}$. Then there exists a scalar $\lambda \neq 0$ in the integral domain $A$ such that

$$
\lambda \varepsilon_{1}, \cdots, \lambda \varepsilon_{n} \in W .
$$

In particular, $\Gamma$ acts irreducibly on the vector space $V$; the representation is in fact absolutely irreducible.

Proof. Not all the images $\left(T_{i}-1\right) W$ can be zero; for that would mean that all the vectors $w$ in $W$ are point-wise fixed by all the $T_{i}$; since distinct eigenspaces of a unitary operator are orthogonal, this means that $w$ is orthogonal to $\varepsilon_{i}$ for each $i$; therefore, $w=0$ since $h$ is nondegenerate.

The image of $\left(T_{i}-1\right)$ consists of multiples of $\varepsilon_{i}$. Therefore, there exists an integer $i$ such that $W$ contains a multiple $\lambda_{i} \varepsilon_{i}$ for some $\lambda_{i} \neq 0$. Since $W$ is stable under all the $T_{j}$, the equation

$$
T_{i-1}\left(\varepsilon_{i}\right)=a_{i-1} \varepsilon_{i}+b_{i-1} \varepsilon_{i-1},
$$

shows that a multiple, namely $b_{i-1} \lambda_{i} \varepsilon_{i-1}=\lambda_{i-1} \varepsilon_{i-1}$ lies in $W, \cdots$, multiples of $\varepsilon_{1}, \cdots, \varepsilon_{i}$ lie in $W$. Similarly, the equation

$$
T_{i-1}\left(\varepsilon_{i}\right)=c_{i+1}\left(\varepsilon_{i+1}\right)+d_{i+1} \varepsilon_{i}, d_{i+1} \neq 0,
$$


shows that a nonzero multiple of $\varepsilon_{i+1}$ lies in $W, \cdots$, a multiple of $\varepsilon_{n}$ lies in $W$. This proves the first part of the lemma.

The foregoing proof also shows the irreducibility for any field $\Omega$ with an involution containing $A$ in its fixed points. Since the fixed field of $\Omega$ under the involution may be embedded in an algebraically closed field $F$, and over $F$ the unitary group becomes $G L_{n}(F)$, it follows that the irreducibility is true in this case as well: the action of $\Gamma$ is absolutely irreducible.

We will now derive a corollary of Lemma 7 which will be used later in the proof of (part [3] of) Proposition [18. We will keep the notation preceding (and including) Lemma 7 . Denote by $\mathbb{Z}[\Gamma]\left(\varepsilon_{i}\right)$ the additive subgroup of $A^{n}$ spanned by the $\Gamma$ translates of the vector $\varepsilon_{i}$.

Corollary 2. Fix $1 \leq i \leq n$. Let $H_{i}$ denote a subgroup of the group $A^{*}$ of units of the integral domain $A$ such that for every $h \in H_{i}$ there exists an element $\gamma \in \Gamma$ such that $h \varepsilon_{i}=\gamma\left(\varepsilon_{i}\right)$.

[1] There exists a $\lambda=\lambda_{i} \neq 0$ in $A$ such that for every $j$, we have $H_{i} \lambda \varepsilon_{j} \subset \mathbb{Z}[\Gamma]\left(\varepsilon_{i}\right)$.

[2] Suppose for each $i, H_{i}$ is as in [1]. Let $H$ be the subgroup of $A^{*}$ generated by $H_{1}, \cdots H_{n}$ (then $H=H_{1} \cdots H_{n}$ is the product). Then there exists a $\lambda \neq 0$ such that for every $h \in H$ and every $j$, the element $\lambda h \varepsilon_{j}$ lies in the $\Gamma$ module generated by $\varepsilon_{1}, \cdots, \varepsilon_{n}$.

Proof. An easy induction shows that [1] implies [2]. We now prove [1].

Fix $i$. By Lemma 7, there exists a nonzero $\lambda \in A$ such that $\lambda \varepsilon_{j} \in$ $\mathbb{Z}[\Gamma]\left(\varepsilon_{i}\right)$. Let $h \in H_{i}$; by assumption, there exists $\gamma \in \Gamma$ such that $\gamma \varepsilon_{i}=h \varepsilon_{i}$. Therefore, $\lambda\left(h \varepsilon_{j}\right)=h\left(\lambda \varepsilon_{j}\right) \in h\left(\mathbb{Z}[\Gamma]\left(\varepsilon_{i}\right)=\mathbb{Z}[\Gamma]\left(h \varepsilon_{i}\right) \subset\right.$ $\mathbb{Z}[\Gamma] \gamma \varepsilon_{i}=\mathbb{Z}[\Gamma]\left(\varepsilon_{i}\right)$.

2.3. Some results on algebraic groups. Let $U \subset S L_{n}(\mathbb{C})$ be the unipotent algebraic group consisting of the set of matrices $u$ of the form

$$
u=\left(\begin{array}{cccc}
1 & x_{2} & \cdots & x_{n} \\
0 & 1 & \cdots & 0 \\
\cdots & \cdots & \cdots & \\
0 & 0 & \cdots & 1
\end{array}\right)
$$

This is the subgroup which preserves the partial flag

$$
0 \subset \mathbb{C} e_{1} \subset \mathbb{C}^{n},
$$


and acts trivially on successive quotients.

Proposition 8. Let $H \subset S L_{n}(\mathbb{C})$ be a reductive algebraic subgroup which contains the unipotent algebraic group $U$. Then $H=S L_{n}(\mathbb{C})$.

Proof. Denote by $T$ the group of diagonals in $S L_{n}$. The Lie algebra of $S L_{n}$ splits into eigenspaces for the action of $T$, and the eigenvectors are $E_{i j}$ and $E_{i i}-E_{j j}$ where $E_{i j}$ is, in the usual notation, the $n \times n$ matrix whose $i j$-th entry is 1 and all other entries are zero. Then the Lie algebra $\mathfrak{u}$ of $U$ is spanned by $E_{1 i}$ with $1<i$.

Let $\mathfrak{h}$ be the Lie algebra of $H$. Write the direct sum decomposition $s l_{n}(\mathbb{C})=\mathfrak{h} \oplus \mathfrak{h}^{\prime}$ as modules under the adjoint action of $H$ (we use the assumption that $H$ is reductive). Since $U$ is unipotent, if $\mathfrak{h}^{\prime}$ is non-zero, there exists an $X \in \mathfrak{h}^{\prime}, X \neq 0$, which is fixed by $U$. This means that the linear transformation $X$ commutes with $U$.

The centraliser $\mathfrak{z}$ of $U$ in $s l_{n}(\mathbb{C})$ is stable under the action of the diagonals $T$, since $U$ is $T$-stable. Hence $\mathfrak{z}$ splits into eigenspaces for $T$. Since $E_{1 i} \in \operatorname{Lie}(U)$, the equation $\left[E_{1 i}, E_{i j}\right]=E_{1 j} \neq 0$ shows that the centraliser $\mathfrak{z}$ cannot contain $E_{i j}$ with $i \geq 2$. It is also clear that the lie algebra of $T$ acts faithfully on $\operatorname{Lie}(U)$ under the adjoint action; therefore,

$$
\mathfrak{z}=\oplus \mathbb{C}_{j \geq 2} E_{1 j}=\operatorname{Lie}(U) \subset \mathfrak{h} .
$$

Hence $X$ must lie in $\mathfrak{h}$; this is impossible and therefore, $\mathfrak{h}^{\prime}=0$.

Let $V$ be an $n$-dimensional vector space over $\mathbb{C}$ and $W, W^{\prime}$ be two distinct codimension one subspaces and suppose we are given a decomposition $V=W \oplus \mathbb{C} v$ and $V=W^{\prime} \oplus \mathbb{C} v^{\prime}$. Assume that $v, v^{\prime}$ are linearly independent over $\mathbb{C}$. We will view $S L(W)$ (resp. $S L\left(W^{\prime}\right)$ ) as the subgroup of elements of $S L(V)$ which stabilise the subspace $W$ (resp. $W^{\prime}$ ) and fix the vector $v$ (resp. $\left.v^{\prime}\right)$.

Lemma 9. $S L(V)$ is generated by $S L(W)$ and $S L\left(W^{\prime}\right)$.

Proof. Put $X=W \cap W^{\prime}$. Then by our assumptions, $X$ has codimension one in both $W$ and $W^{\prime}$. Fix a vector $w \in W$ (resp. $w^{\prime} \in W^{\prime}$ ) which does not lie in $X$. We have the decomposition $W=X \oplus \mathbb{C} w$ and $W^{\prime}=X \oplus \mathbb{C} w^{\prime}$. Write $E=\mathbb{C} w \oplus \mathbb{C} w^{\prime}$. Then $\operatorname{sl}(V)=\operatorname{sl}(X) \oplus$ $\left(X \otimes E^{*}\right) \oplus\left(X^{*} \otimes E\right) \oplus \operatorname{sl}(E) \oplus Y$. Here $Y$ is the space of trace zero endomorphisms of $V$ which act by a scalar on $X$ and by a scalar on $E$. 
If $h$ is the sub-algebra generated by $\operatorname{sl}(W)$ and $\operatorname{sl}\left(W^{\prime}\right)$, then $h$ (in fact the subspace $\left.\operatorname{sl}(W)+\operatorname{sl}\left(W^{\prime}\right)\right)$ contains $E \otimes X$ and $X^{*} \otimes E$ as subspaces; the sub-algebra generated by these subspaces contains $\operatorname{sl}(E)$ (it is easy to capture the remaining one dimensional space $Y$ in the subalgebra $h)$. Therefore $h=s l(V)$.

2.4. Products. The following Lemma is proved in [25] and will be used in deducing the arithmeticity of the monodromy in Theorem 1 from the arithmeticity of the images of the Gassner representation at roots of unity (Theorem [16). After it was obtained, we learnt that this was already proved (in roughly the same form) in [14] and in [10].

Suppose $X$ is a finite indexing set and for each element $p \in X$, let $K_{p}$ be a number field and $G_{p}$ be an absolutely almost simple group defined over $K_{p}$ with $\infty-\operatorname{rank}\left(G_{e}\right) \geq 2$. We assume that if $e, f \in X$ are distinct elements of $X$, then either $K_{e}$ and $K_{f}$ are not isomorphic as number fields or $G_{e}$ and $G_{f}$ are not isomorphic as algebraic groups over $K_{e} \simeq K_{f}$ (both groups thought of as algebraic groups over the same field $K_{e} \simeq K_{f}$ ).

Lemma 10. With the preceding assumptions, suppose $\Gamma \subset \prod_{e \in X} G_{e}\left(O_{e}\right)$ is a subgroup of a product of higher rank arithmetic groups. Assume that for each $e \in X$, the projection of $\Gamma$ in $G_{e}\left(O_{e}\right)$ has finite index in $G_{e}\left(O_{e}\right)$. Then $\Gamma$ has finite index in the product.

\section{Action of the Braid Group on a Free Group}

In this section, we first recall an action of the braid group on a free group, defined by Artin (see 3.2). This gives an action of the pure braid group on the first integral homology of the commutator subgroup of the free group. This action of the pure braid group is closely related to the Gassner representation. To make this relation precise, we need to replace the free group with the free product $F$ of the free group with its abelianisation. There is an action of the braid group on the kernel of the natural homomorphism from $F$ onto the abelianisation of the free group. The resulting action of the pure braid group on the homology of this kernel is identified with the Gassner representation (see 3.4). We can then define the reduced Gassner representation and construct a convenient basis $\left\{\varepsilon_{i}: 1 \leq i \leq n\right\}$ for it; we will use this basis in the next section to specialise the reduced Gassner representation at roots of unity. 
3.1. The pure braid group. The braid group $B_{n+1}$ on $n+1$ strands is the free group on the generators $s_{1}, s_{2}, \cdots, s_{n}$ modulo the relations

$$
s_{i} s_{j}=s_{j} s_{i}(|i-j| \geq 2) \text {, and } s_{i} s_{j} s_{i}=s_{j} s_{i} s_{j}(|i-j|=1) \text {. }
$$

The symmetric group $S_{n+1}$ on $n+1$ symbols is the free group on the generators $\sigma_{1}, \cdots, \sigma_{n}$ modulo the relations

$$
\sigma_{i} \sigma_{j}=\sigma_{j} \sigma_{i}(|i-j| \geq 2), \sigma_{i} \sigma_{j} \sigma_{i}=\sigma_{j} \sigma_{i} \sigma_{j}(|i-j|=1),
$$

and the additional relations $\sigma_{i}^{2}=1$.

There is a natural surjective homomorphism $B_{n+1} \rightarrow S_{n+1}$ of the braid group onto the symmetric group on $n+1$ letters given by $s_{i} \mapsto \sigma_{i}$. The kernel of this homomorphism is the "Pure Braid Group" $P_{n+1}$ on $n+1$ strands. The elements $s_{i}^{2}$ of $B_{n+1}$ lie in $P_{n+1}$. It can easily be shown that the conjugates of these elements $s_{i}^{2}$ under all the elements of $B_{n+1}$ generate $P_{n+1}$. If $i<j$ denote by $\Pi_{i j}$ the product in $B_{n+1}$ given by $\Pi_{i j}=s_{i+1} s_{i+2} \cdots s_{j-1}$. For $r<s$, Set $A_{r s}=\Pi_{r s}^{-1} s_{r}^{2} \Pi_{r s}$. In particular, $A_{r, r+1}=s_{r}^{2}$. The pure braid group is in fact generated by the elements $A_{r s}$.

3.2. Artin's Theorem. Let $F_{n+1}$ be the free group on $n+1$ generators $x_{1}, \cdots, x_{n+1}$. The braid group $B_{n+1}$ acts ([6], page 21, Corollary (1.8.3)) on the free group $F_{n+1}$ as follows.

$$
\begin{gathered}
s_{i}\left(x_{j}\right)=x_{j} \text { if } j \neq i, i+1, \\
s_{i}\left(x_{i}\right)=x_{i} x_{i+1} x_{i}^{-1} \text { and } s_{i}\left(x_{i+1}\right)=x_{i} .
\end{gathered}
$$

The following Theorem of Artin is fundamental to the rest of the section.

Theorem 11. The above formulae give an action of the braid group on $F_{n+1}$; moreover, the action is faithful.

The action of $B_{n+1}$ is such that on the abelianisation $\mathbb{Z}^{n+1}$ of $F_{n+1}$, the action is by the symmetric group $S_{n+1}$ and the kernel of the map $B_{n+1} \rightarrow S_{n+1}$ is the pure braid group $P_{n+1}$. .

The action of the generators $A_{r, s}$ of the pure braid group $P_{n+1}$ can be worked out (from these formulae for the action of $s_{i}$ ) ([6], p. 25, Corollary 1.8.3)):

$$
\begin{gathered}
A_{r, s}\left(x_{i}\right)=x_{i}(i<r \text { or } i>s), \quad A_{r, s}\left(x_{r}\right)=\left(x_{r} x_{s}\right) x_{r}\left(x_{r} x_{s}\right)^{-1}, \\
A_{r, s}\left(x_{s}\right)=x_{r} x_{s} x_{r}^{-1}, \quad A_{r, s}\left(x_{i}\right)=\left[x_{r}, x_{s}\right] x_{i}\left[x_{r}, x_{s}\right]^{-1}(r<i<s) .
\end{gathered}
$$

In particular, each generator $x_{i}$ of $F_{n+1}$ goes into a conjugate of itself under the action of $P_{n+1}$. 
3.3. Action on Certain Subgroups and Sub-quotients. Suppose $F$ is a group, and $Q$ a quotient of $F$ and $K$ the kernel of the quotient map $F \rightarrow Q$. Then there is the exact sequence

$$
1 \rightarrow K \rightarrow F \rightarrow Q \rightarrow 1
$$

Denote by $K^{1}=[K, K]$ the commutator subgroup of $K$ and by $K^{a b}=$ $K / K^{1}$ the abelianisation of $K$. Then the conjugation action of $F$ stabilises $[K, K]$ and $F$ acts on $K^{a b}$. We may write $K^{a b}$ additively. The action of $F$ on $K^{a b}$ is such that $K$ acts trivially; hence the action of $F$ on $K^{a b}$ descends to an action of $Q$ on $K^{a b}$ and hence $K^{a b}$ becomes a $\mathbb{Z}[Q]$-module where $\mathbb{Z}[Q]$ is the group ring of $Q$ with $\mathbb{Z}$-coefficients.

We have an exact sequence

$$
0 \rightarrow K^{a b} \rightarrow F / K^{1} \rightarrow Q \rightarrow 1 .
$$

Suppose $H \subset A u t(F)$ be a subgroup of the automorphism group of $F$ such that $H$ stabilises $K$ and acts trivially on $Q$; then $H$ acts on the foregoing exact sequence and the action of $H$ on $K^{a b}$ commutes with the action of $Q$ on $K^{a b}$; therefore, $H$ acts by $\mathbb{Z}[Q]$-module maps on the $Q$ module $K^{a b}$.

3.4. The Gassner Representation. In the notation of subsection (3.3), we take $F=F_{n+1} * F_{n+1}^{a b}$ to be the free product of the group $F_{n+1}$ and its abelianisation $F_{n+1}^{a b}$ (written multiplicatively). Write, temporarily, $H$ for $F_{n+1}$. The abelianisation of $F$ is $H^{a b} \times H^{a b}$. There is the multiplication map $m: H^{a b} \times H^{a b}$ given by $(x, y) \mapsto x y$. We have the composite map $\phi: F=H * H^{a b} \rightarrow H^{a b} \times H^{a b} \stackrel{m}{\rightarrow} H^{a b}$. This is a surjection with kernel $K$, say. We then have a split exact sequence

$$
1 \rightarrow K \rightarrow H * H^{a b} \rightarrow H^{a b} \rightarrow 1 \text {. }
$$

The group in the middle is then a semi-direct product $H * H^{a b} \simeq$ $K \rtimes H^{a b}$, since the exact sequence splits. Write the elements of the semi-direct product as a pair $(w, t)$ with $w \in K$ and $t \in H^{a b}$. Write the image of the standard generators $x_{i}$ of $H$ in this semi-direct product group as a pair $x_{i}=\left(y_{i}, X_{i}\right)$. Therefore $y_{i}$ and $X_{j}$ generate the group $F$ and hence the $y_{i}$ generate $K$ as a normal subgroup of $F$.

As in subsection 3.3, we take the quotient of the group $F$ by the commutator subgroup $[K, K]$, and get an exact sequence

$$
0 \rightarrow K^{a b} \rightarrow \frac{H * H^{a b}}{[K, K]} \rightarrow H^{a b} \rightarrow 1
$$


which is still split over $H^{a b}$. Hence we may write the group in the middle as a semi-direct product $F /[K, K]=K^{a b} \rtimes H^{a b}$. An element of this group is written as a pair $(w, t)$ with $w \in K^{a b}$ and $t \in H^{a b}$; the conjugation by $t$ on $K^{a b}$ is simply multiplying by the element $t$, when we view $K^{a b}$ as a module over the group ring $\mathbb{Z}\left[H^{a b}\right]$. We write $H^{a b}=F_{n+1}^{a b}$ multiplicatively in the form $H^{a b}=X_{1}^{\mathbb{Z}} X_{2}^{\mathbb{Z}} \cdots X_{n+1}^{\mathbb{Z}}$. Denote by $e_{i}$ the image of $y_{i} \in K$ in the abelianisation $K^{a b}$. By the conclusion of the last paragraph, the elements $e_{i}$ generate $K^{a b}$ as a module over the group ring $\mathbb{Z}\left[H^{a b}\right]$ :

$$
K^{a b}=\sum_{i=1}^{n+1} \mathbb{Z}\left[H^{a b}\right]\left(e_{i}\right) .
$$

We will now show that $K^{a b}$ is a free module over $R$ with $e_{i}$ as basis. Write $R=\mathbb{Z}\left[H^{a b}\right]$. We will view $R$ as a module over the multiplicative group $H^{a b}$ by the formula $x\left(f_{1}, \cdots, f_{n+1}\right)=\left(x f_{1}, \cdots, x f_{n+1}\right)$ where $x \in H^{a b}$ is viewed as a unit in $R$. Let $\left(\xi_{i}\right)_{i \leq n+1}$ be the standard basis of $R^{n+1}$. Form the semi-direct product $\mathcal{H}=R^{n+1} \rtimes H^{a b}$. We then get a homomorphism from the free product $H * H^{a b}$ into $\mathcal{H}$ by specifying the homomorphism on the generators $x_{i} \mapsto\left(\xi_{i}, X_{i}\right)$ and $t \mapsto(0, t) \in \mathcal{H}=$ $R^{n+1} \rtimes F_{n+1}^{a b}$. Then we get a homomorphism $H * H^{a b}$ which takes $y_{i}$ to the element $\xi_{i}$. Therefore, we get a homomorphism of $R$ modules from $K^{a b}$ into $R^{n+1}$ which sends $e_{i}$ into the basis element $\xi_{i}$. This shows that the $e_{i}$ are linearly independent over $R$; the last line of the preceding paragraph tells us that the $e_{i}$ span $K^{a b}$. Hence the $e_{i}$ form a basis of $K^{a b}$ and

$$
K^{a b}=R^{n+1}=\bigoplus_{i=1}^{n+1} R e_{i}
$$

We now write the product of two elements $x=(w, t), y=\left(w^{\prime}, t^{\prime}\right) \in$ $\bar{F}_{n+1}=K^{a b} \rtimes H^{a b}$. The product is given by

$$
x y=(w, t)\left(w^{\prime}, t^{\prime}\right)=\left(w+t w^{\prime} t^{-1}, t t^{\prime}\right)=\left(w+t\left(w^{\prime}\right), t t^{\prime}\right) .
$$

The inverse of $x=(w, t)$ is $x^{-1}=\left(-t^{-1} w, t^{-1}\right)$. An easy induction shows that

$$
\left(v_{1}, t_{1}\right)\left(v_{2}, t_{2}\right) \cdots\left(v_{n+1}, t_{n+1}\right)=\left(\sum_{i=1}^{n+1} t_{1} t_{2} \cdots t_{i-1} v_{i}, t_{1} t_{2} \cdots t_{n+1}\right) .
$$

In this formula, $v_{i}$ are vectors in $K^{a b}$ and $K^{a b}$ is viewed as an $R$ module. The following lemma is an immediate consequence of these formulae and the formulae in 3.2 . 
Lemma 12. Let $x_{r}, x_{s} \in F_{n+1}=H$ be as before and $\left(e_{r}, X_{r}\right)=$ $\bar{x}_{r},\left(e_{s}, X_{s}\right)=\bar{x}_{s} \in \bar{F}=F /[K, K]$ be their images in the quotient group $F /[K, K]$ (which is a semi-direct product). Then we have the formulae (read in $\bar{F})$

$$
\begin{gathered}
\overline{x_{r} x_{s} x_{r}^{-1}}=\left(e_{r}+X_{r} e_{s}-X_{s} e_{r}, X_{s}\right)=\left(\left(1-X_{s}\right) e_{r}+X_{r} e_{s}, X_{s}\right), \\
x_{r} x_{s} x_{r} x_{s}^{-1} x_{r}^{-1}=\left(e_{r}+X_{r}\left(e_{s}\right)+X_{r} X_{s}\left(e_{r}\right)-X_{r}^{2}\left(e_{s}\right)-X_{r} e_{r}, X_{r}\right), \\
\overline{\left[x_{r}, x_{s}\right]}=\left(1-X_{s}\right) e_{r}-\left(1-X_{r}\right) e_{s} \text { and } \\
\overline{\left[x_{r}, x_{s}\right] x_{i}\left[x_{r}, x_{s}\right]^{-1}}=\left(e_{i}+\left(1-X_{i}\right) v_{r, s}, X_{i}\right)= \\
=\left(e_{i}+\left(1-X_{i}\right)\left(1-X_{s}\right) e_{r}-\left(1-X_{i}\right)\left(1-X_{r}\right) e_{s}, X_{i}\right) .
\end{gathered}
$$

The braid group $B_{n+1}$ acts on the free group $F_{n+1}$ and hence acts naturally on the free product $F=F_{n+1} * F_{n+1}^{a b}$. The preceding map $F \rightarrow F^{a b}=H^{a b} \times H^{a b} \rightarrow H^{a b}$ is equivariant for the action of $B_{n+1}$ (and $B_{n+1}$ acts via the finite group $S_{n+1}$ on the abelianisation $F_{n+1}^{a b}$ ). Hence $B_{n+1}$ acts on the exact sequence

$$
1 \rightarrow K \rightarrow F \rightarrow H^{a b} \rightarrow 1,
$$

and the pure braid group $P_{n+1}$ acts trivially on $F_{n+1}^{a b}=H^{a b}$. We are therefore in the situation of subsection (3.3), and hence, as in subsection (3.3), the group $P_{n+1}$ acts by $\mathbb{Z}\left[F_{n+1}^{a b}\right]=R$-module maps on $K^{a b}$. We can now compute the action of the standard generators $A_{r, s}$ of the pure braid group $P_{n+1}$ on the images of $x_{i}$ in the quotient group $F /[K, K]$.

[1] Recall that $A_{r, s}\left(x_{i}\right)=x_{i}$ if $i \leq r-1$ or $i \geq s+1$. From Lemma 12, it follows that $A_{r, s}\left(e_{i}\right)=e_{i}$ for these $i$.

[2] $A_{r, s}\left(x_{r}\right)={ }^{x_{r} x_{s}}\left(x_{r}\right)$. When this equation is read modulo $[K, K]$, we see from Lemma 12 that

$$
A_{r, s}\left(e_{r}\right) X_{r}=A_{r, s}\left(e_{r} X_{r}\right)=A_{r, s}\left(x_{r}\right)=e_{r} X_{r} e_{s} X_{s}\left(e_{r} X_{r}\right) X_{s}^{-1} e_{s}^{-1} X_{r}^{-1} e_{r}^{-1} .
$$

Cancelling $X_{r}$ on the right on the left most and right most sides of this equation, we see that

$$
A_{r, s}\left(e_{r}\right)=\left(1-X_{r}+X_{r} X_{s}\right) e_{r}+X_{r}\left(1-X_{r}\right) e_{s} .
$$

[3] The equation $A_{r, s}\left(x_{s}\right)={ }^{x_{r}}\left(x_{s}\right)$ becomes, modulo the subgroup $[K, K]$, the equation

$$
A_{r, s}\left(e_{s}\right) X_{s}=\left(\left(1-X_{s}\right) e_{r}+X_{r} e_{s}\right) X_{s} .
$$


[4] If $r<i<s$ then by Lemma 12,

$A_{r, s}\left(e_{i}\right) X_{i}=A_{r, s}\left(x_{i}\right)={ }^{\left[x_{r}, x_{s}\right]}\left(x_{i}\right)=\left(e_{i}+\left(1-X_{i}\right)\left(\left(1-X_{s}\right) e_{r}-\left(1-X_{r}\right) e_{s}\right)\right) X_{i}$

or

$$
A_{r, s}\left(e_{i}\right)=e_{i}+\left(1-X_{i}\right)\left(\left(1-X_{s}\right) e_{r}-\left(1-X_{r}\right) e_{s}\right) .
$$

These equations imply that with respect to the basis $e_{i}$ of $K^{a b}=$ $R^{n+1}$, the action by $P_{n+1}$ on the $R$ module $K^{a b}$ is exactly the Gassner representation $G_{n}(X): P_{n+1} \rightarrow G L_{n+1}(R)$ (see [6], p 119, formulae $(3-24))$.

Notation. The $\operatorname{ring} R=\mathbb{Z}\left[X_{1}^{ \pm 1}, \cdots, X_{n+1}^{ \pm 1}\right]$ of Laurent polynomials in $n+1$ variables with integral coefficients, is an integral domain. Let $\Omega=\mathbb{Q}\left(X_{1}, \cdots, X_{n+1}\right)$ be its field of fractions. We have the free $R$ module $K^{a b}=\sum_{i=1}^{n+1} R e_{i}$. This may be thought of as an $R$ submodule of the $\Omega$ vector space $K^{a b} \otimes_{R} \Omega=\sum_{i=1}^{n+1} \Omega e_{i}$. We may write $e_{i}=\left(1-X_{i}\right) v_{i}$ for some vector $v_{i} \in K^{a b} \otimes \Omega$.

Now the full braid group $B_{n+1}$ acts on $R$ via $S_{n+1}$ by permuting the indices $X_{i}$ of the generators (and the pure braid group acts trivially). Hence $B_{n+1}$ acts on $\Omega$ by field automorphisms. We denote this action, for $g \in B_{n+1}$ and $\lambda \in \Omega$, by $(g, \lambda) \mapsto g(\lambda)$. The action of $B_{n+1}$ on $K^{a b} \otimes \Omega$ is not linear over $\Omega$ but is "twisted linear": if $\lambda \in \Omega, g \in B_{n+1}$ and $w \in K^{a b}$, then $g(\lambda w)=g(\lambda) g(w)$.

Lemma 13. Let $v_{i}=\frac{1}{1-X_{i}} e_{i}$ with $e_{i}$ and $R$ as before. The $R$ module $\bigoplus_{i=1}^{n+1} R v_{i}$ is stable under the action of $P_{n+1}$.

Proof. Since $P_{n+1}$ acts by $\Omega$-linear maps, it suffices to show that for every $g \in P_{n+1}$ and every $v_{i}$ the translate $g\left(v_{i}\right)$ is an $R$ linear combination of the $v_{j}$. We will in fact prove more; we will show that for every generator $s_{i}$ of the full braid group $B_{n+1}$, the translate $s_{i}\left(v_{j}\right)$ is an $R$ linear combination of the $v_{k}$. The group $B_{n+1}$ acts by twisted $\Omega$ linear maps as before and not by $\Omega$ linear maps; however, it takes an element $\lambda w \in K^{a b}$, with $\lambda \in R$ and $w \in K^{a b}$ into an element of the form $\mu g(w)$ and hence preserves the space $\sum R v_{i}$ provided each $v_{j}$ is mapped into an $R$ linear combination of the $v_{k}$. We now need only check that for each generator $s_{i}$ of $B_{n+1}$ and each $v_{j}$, the translate $s_{i}\left(v_{j}\right)$ is an $R$ linear combination of the vectors $v_{1}, \cdots, v_{n+1}$.

Suppose $j \neq i, i+1$. We have $s_{i}\left(x_{j}\right)=x_{j}$ for $j \neq i, i+1$. Therefore, $s_{i}\left(X_{j}\right)=X_{j}$. Since $x_{i}=\left(e_{i}, X_{i}\right)$ it follows that $s_{i}\left(e_{j}\right)=e_{j}$. We now 
write $e_{j}=\left(1-X_{j}\right) v_{j}$ and note that $s_{i}$ acts trivially on $X_{j}$. Hence

$$
\begin{gathered}
\left.\left(1-X_{j}\right) v_{j}=e_{j}=s_{i}\left(e_{j}\right)=s_{i}\left(1-X_{j}\right) v_{j}\right)= \\
=\left(1-s_{i}\left(X_{j}\right)\right) s_{i}\left(\left(v_{j}\right)=\left(1-X_{j}\right) s_{i}\left(v_{j}\right) .\right.
\end{gathered}
$$

This shows that $s_{i}\left(v_{j}\right)=v_{j}$.

Suppose $j=i$. Then $s_{i}\left(x_{i}\right)=x_{i} x_{i+1} x_{i}^{-1}=\left[x_{i}, x_{i+1}\right] x_{i+1}$. We have expressed a commutator in terms of the $e_{j}$ (see (4) of Lemma 12): hence the commutator $\left[x_{i}, x_{i+1}\right]=\left(1-X_{i+1}\right) e_{i}-\left(1-X_{i}\right) e_{i+1}$. Therefore,

$$
\left(s_{i}\left(e_{i}\right), X_{i+1}\right)=s_{i}\left(\left(e_{i}, X_{i}\right)\right)=\left(\left(1-X_{i+1}\right) e_{i}-\left(1-X_{i}\right) e_{i+1}+e_{i+1}, X_{i+1}\right) .
$$

Comparing the extreme left and right hand sides of this equation, we see that $s_{i}\left(e_{i}\right)=\left(1-X_{i+1}\right) e_{i}+X_{i} e_{i+1}$. Now write $e_{i}=\left(1-X_{i}\right) v_{i}$ and similarly for $e_{i+1}$. Then we have

$$
\begin{gathered}
\left(1-X_{i+1}\right) s_{i}\left(v_{j}\right)=s_{i}\left(\left(1-X_{i}\right) v_{i}\right)=s_{i}\left(e_{i}\right)=\left(1-X_{i+1}\right) e_{i}+X_{i} e_{i+1}= \\
=\left(1-X_{i+1}\right)\left(1-X_{i}\right) v_{i}+X_{i}\left(1-X_{i+1}\right) v_{i+1} .
\end{gathered}
$$

Cancelling $\left(1-X_{i+1}\right)$ on both the extreme right and left hand sides of this equation, we get

$$
s_{i}\left(v_{i}\right)=\left(1-X_{i}\right) v_{i}+X_{i} v_{i+1} .
$$

Suppose $j=i+1$. Then $s_{i}\left(x_{i+1}\right)=x_{i}$. Reading this as in Lemma 12 we get $s_{i}\left(e_{i+1}, X_{i+1}\right)=\left(e_{i}, X_{i}\right)$ and $s_{i}\left(X_{i+1}\right)=X_{i}$. Therefore, we get $s_{i}\left(e_{i+1}\right)=e_{i}$. Writing $e_{i}\left(1-X_{i}\right) v_{i}$ we see that

$$
\left(1-X_{i}\right) s_{i}\left(v_{i+1}\right)=s_{i}\left(\left(1-X_{i+1}\right) v_{i+1}\right)=s_{i}\left(e_{i+1}\right)=e_{i}=\left(1-X_{i}\right) v_{i} .
$$

Comparing the extreme right and left hand sides of this equation, we get

$$
s_{i}\left(v_{i+1}\right)=v_{i} .
$$

From the last three paragraphs, we see that each $s_{i}\left(v_{j}\right)$ is an $R$-linear combination of the $v_{j}$. Therefore, the lemma follows.

Lemma 14. Set $\varepsilon_{i}=v_{i}-v_{i+1}$ for $1 \leq i \leq n$. Then the $R$ module generated by $\left\{\varepsilon_{i}: 1 \leq i \leq n\right\}$ is stable under the action of $P_{n+1}$.

In particular, with respect to the basis $\varepsilon_{1}, \cdots, \varepsilon_{n}$ the transformation $T_{i}=s_{i}^{2}$ has the matrix form

$$
\left(\begin{array}{ccc}
1 & 0 & 0 \\
X_{i}\left(1-X_{i+1}\right) & X_{i} X_{i+1} & 1-X_{i} \\
0 & 0 & 1
\end{array}\right) \oplus 1_{n-3}
$$


where the $3 \times 3$ matrix is with respect to the basis elements $\varepsilon_{i-1}, \varepsilon_{i}, \varepsilon_{i+1}$ and $s_{i}^{2}$ acts as identity on the basis elements $\varepsilon_{j}$ for the other indices $j$. In particular, $s_{i}^{2}$ are complex reflections.

Proof. As in the proof of Lemma 13, because the full braid group acts by "twisted" $\Omega$ linear maps on $K^{a b} \otimes \Omega$, it suffices to check that the full braid group preserves the $R$ module spanned by the $\varepsilon_{i}$.

We use the formulae in the proof of Lemma 13. Suppose $j+1<i$. Then

$$
s_{i}\left(\varepsilon_{j}\right)=s_{i}\left(v_{j}-v_{j+1}\right)=v_{j}-v_{j+1}=\varepsilon_{j} .
$$

Similarly, $s_{i}\left(\varepsilon_{j}\right)=\varepsilon_{j}$ if $j>i+1$. We now get from the formulae for $s_{i}\left(v_{i}\right)$ and $s_{i}\left(v_{i+1}\right)$ obtained from Lemma 13, that

$$
\begin{gathered}
s_{i}\left(\varepsilon_{i}\right)=s_{i}\left(v_{i}-v_{i+1}\right)=\left(1-X_{i}\right) v_{i}+X_{i} v_{i+1}-v_{i}=-X_{i} \varepsilon_{i}, \\
s_{i}\left(\varepsilon_{i+1}\right)=s_{i}\left(v_{i+1}-v_{i+2}\right)=v_{i}-v_{i+2}=\varepsilon_{i}+\varepsilon_{i+1} .
\end{gathered}
$$

Finally,

$$
\begin{gathered}
s_{i}\left(\varepsilon_{i-1}\right)=s_{i}\left(v_{i-1}-v_{i}\right)=v_{i-1}-\left(1-X_{i}\right) v_{i}-X_{i} v_{i+1}= \\
=\varepsilon_{i-1}+X_{i} \varepsilon_{i} .
\end{gathered}
$$

This proves the first part of the lemma.

To prove the second part, we must compute $s_{i}^{2}\left(\varepsilon_{j}\right)$. If $j+1<i$ or if $j>i+1$ then $s_{i}\left(\varepsilon_{j}\right)=\varepsilon_{j}$; hence $s_{i}^{2}\left(\varepsilon_{j}\right)=\varepsilon_{j}$. We now compute $s_{i}^{2}\left(\varepsilon_{i-1}\right)$ :

$$
\begin{aligned}
& s_{i}\left(s_{i}\left(\varepsilon_{i-1}\right)\right)=s_{i}\left(\varepsilon_{i-1}+X_{i} \varepsilon_{i}\right)=s_{i}\left(\varepsilon_{i-1}\right)+X_{i+1} s_{i}\left(\varepsilon_{i}\right)= \\
& =\varepsilon_{i-1}+X_{i} \varepsilon_{i}+X_{i+1}\left(-X_{i} \varepsilon_{i}\right)=\varepsilon_{i-1}+X_{i}\left(1-X_{i+1}\right) \varepsilon_{i} .
\end{aligned}
$$

Next we compute

$$
s_{i}^{2}\left(\varepsilon_{i}\right)=s_{i}\left(-X_{i} \varepsilon_{i}\right)=-X_{i+1} s_{i}\left(\varepsilon_{i}\right)=X_{i+1} X_{i} \varepsilon_{i} .
$$

Finally,

$$
s_{i}^{2}\left(\varepsilon_{i+1}\right)=s_{i}\left(\varepsilon_{i}+\varepsilon_{i+1}\right)=-X_{i} \varepsilon_{i}+\varepsilon_{i}+\varepsilon_{i+1}=\left(1-X_{i}\right) \varepsilon_{i}+\varepsilon_{i+1} .
$$


3.5. An invariant element in the Gassner representation. The product element $x_{1} x_{2} \cdots x_{n+1} \in F_{n+1}$ is invariant under the action of the braid group $B_{n+1}$. The image of $x_{1} x_{2} \cdots x_{n+1}$ in the semi-direct product group $F /[K, K]$ is therefore invariant; since the image of $x_{i}$ is written as $\left(e_{i}, X_{i}\right)$, it follows from the formulae before Lemma 12 that

$$
\begin{gathered}
x_{1} x_{2} \cdots x_{n+1}=\left(e_{1} X_{1}\right)\left(e_{2} X_{2}\right) \cdots\left(e_{n+1} X_{n+1}\right)= \\
=\left(e_{1}+X_{1}\left(e_{2}\right)+X_{1} X_{2}\left(e_{3}\right)+\cdots+X_{1} X_{2} \cdots X_{n}\left(e_{n+1}\right), X_{1} X_{2} \cdots X_{n+1}\right) .
\end{gathered}
$$

Therefore, the element

$$
v=\sum_{i=1}^{n+1} X_{1} X_{2} \cdots X_{i-1}\left(e_{i}\right) \in K^{a b}
$$

is invariant under the action of the pure braid group. Moreover, since all the coefficients $X_{1} X_{2} \cdots X_{i}$ of $v$ are units in the ring

$$
R=\mathbb{Z}\left[X_{1}^{ \pm 1}, X_{2}^{ \pm 1}, \cdots X_{n+1}^{ \pm 1}\right]
$$

it follows that $v$ is part of a basis of $K^{a b}=\oplus_{i=1}^{n+1} R e_{i}=R^{n+1}$. Consider the quotient module $V_{n}(X)=K^{a b} / R v$. Then $V_{n}(X) \simeq R^{n}$ and is a module over $P_{n+1}$. Let $\Omega$ be the field of fractions of the integral domain $R$. Then $V_{n}(X) \otimes_{R} \Omega$ is called the reduced Gassner Representation over $\Omega$ the field of fractions, and is denoted

$$
g_{n}(X): P_{n+1} \rightarrow G L_{n}(\Omega) .
$$

3.6. A supplement to the space of invariants. We will now find a sub-module $W_{n}(X)$ which has zero intersection with the space $R v$ of multiples of the invariant vector $v$ in the (non-reduced) Gassner representation, which is stable under the action of the pure braid group $P_{n+1}$ and is free of rank $n$ over $R$. We will view $K^{a b}=\oplus_{i=1}^{n+1} R e_{i}$ as a subgroup of the $\Omega$ vector space $\Omega^{n+1}=K^{a b} \otimes_{R} \Omega=\oplus_{i=1}^{n+1} \Omega e_{i}$. Write $e_{i}=\left(1-X_{i}\right) v_{i}$, with $v \in \Omega^{n+1}$.

The calculations of Lemma 12 show that the $R$-module $L=\oplus_{i=1}^{n+1} R v_{i}$ is stable under the action of the pure braid group $P_{n+1}$. The lemma also implies that the free $R$ sub-module

$$
W=\oplus_{i=1}^{n} R \varepsilon_{i} \simeq R^{n},
$$

(where $\varepsilon_{i}=v_{i}-v_{i+1}$ ) is stable under $P_{n+1}$. We note that the commutator $\left[x_{i}, x_{i+1}\right]$, (see equation 4 of Lemma [12) viewed as an element of the kernel $K^{a b}$ has the form

$\left[x_{i}, x_{i+1}\right]=\left(1-X_{i}\right) e_{i+1}-\left(1-X_{i+1}\right) e_{i}=\left(1-X_{i}\right)\left(1-X_{i+1}\right)\left(v_{i}-v_{i+1}\right)=$ 
i.e.

$$
\left[x_{i}, x_{i+1}\right]=\left(1-X_{i}\right)\left(1-X_{i+1}\right) \varepsilon_{i} .
$$

As an $R$ module, $W$ is a summand of $L: L=W \oplus R v_{n+1}$ (since $\varepsilon_{i}=v_{i}-v_{i+1}$ form a basis of $W$ ). It can be proved that $W$ is not a direct summand as a module over $P_{n+1}$; however, it is so, when all the modules are tensored with the field $\Omega$ :

$$
L \otimes \Omega=\Omega^{n+1}=W \otimes \Omega \oplus \Omega v,
$$

where $v=\sum_{i=1}^{n+1} X_{1} \cdots X_{i-1} e_{i}$. Write $\pi=X_{1} \cdots X_{i}$ and $e_{i}=\left(1-X_{i}\right) v_{i}$ as before. Then the invariant element $v$ of equation 7 can be written as a linear combination of $\varepsilon_{i}$ and $e_{n+1}$ :

$$
v=\left(1-\pi_{1}\right) \varepsilon_{1}+\left(1-\pi_{2}\right) \varepsilon_{2}+\cdots\left(1-\pi_{n}\right) \varepsilon_{n}+\left(1-\pi_{n+1}\right) v_{n+1} \in L
$$

We will refer to the $P_{n+1}$ module $W=\oplus R \varepsilon_{i} \simeq R^{n}$ as the reduced

Gassner representation over the ring $R$. We will later consider the reduction (modulo ideals of $R$ ) of $W$ to obtain specialisations of the reduced Gassner representation. Over the fraction field $\Omega$, the representation $W \otimes \Omega$ is isomorphic to the quotient $K^{a b} \otimes \Omega / \Omega v$ and hence the terminology is consistent with the end of the preceding subsection.

\section{Properties of the The Gassner Representation}

In this section, we prove some properties of the Gassner representation and its specialisations. We first construct (in subsection 4.1) a skew Hermitian form on the reduced Gassner representation, which is invariant under the action of the pure braid group. The existence of the form is due to [12] (see also [8]), but the construction for the basis $\varepsilon_{i}$ defined in the previous section, may perhaps be new. Using this form, it is easy to decide when specialisations of the Gassner representation (especially $d$-th roots of unity) are irreducible.

It turns out that the specialised reduced Gassner representation is irreducible if and only if the form is nondegenerate. When it is degenerate, we will get, in the image of the Gassner representation, many unipotent elements (Proposition 19). This is crucial to our proof of arithmeticity (Theorem [16).

\subsection{A (skew) hermitian form preserved by the pure braid}

group. It is known (see [12] Theorem (3.3)) that the Gassner representation has a skew-hermitian form invariant under the action of the pure braid group. To compute this form with respect to the " $\varepsilon$ "-basis $\left\{\varepsilon_{i}: 1 \leq i \leq n\right\}$ of the reduced Gassner representation, we proceed as follows. Since the matrices $s_{i}^{2}$ act by complex reflections, and $\varepsilon_{i}$ is the 
unique (up to scalar multiples) eigenvector with eigenvalue $X_{i} X_{i+1} \neq 1$, it follows from Lemma 7 that the group generated by the $s_{i}^{2}$ acts already irreducibly on $R^{n} \otimes \Omega$, where $\Omega$ is the field of fractions of $R$. Further, since $s_{i}^{2}$ are unitary, the eigenvectors with eigenvalue $=1$ of $s_{i}^{2}$ are orthogonal with respect to $h$, to the eigenvector $\varepsilon_{i}$. Hence $\varepsilon_{j}$ and $\varepsilon_{i}$ are orthogonal if $|i-j| \geq 2$.

The ring $R$ has an involution given by $X_{i} \mapsto X_{i}^{-1}=Y_{i}$. Since our form is to be skew hermitian, we have that $h\left(\varepsilon_{1}, \varepsilon_{1}\right)$ is an element of $R$ which is "imaginary" (i.e. it goes to its negative under the involution). We normalise it so that

$$
h\left(\varepsilon_{1}, \varepsilon_{1}\right)=\frac{1-X_{1} X_{2}}{\left(1-X_{1}\right)\left(1-X_{2}\right)} .
$$

Consider the element $h\left(\varepsilon_{1}, \varepsilon_{2}\right)$; the invariance of $h$ under forces the equation

$$
h\left(\varepsilon_{1}, \varepsilon_{2}\right)=h\left(s_{1}^{2}\left(\varepsilon_{1}\right), s_{1}^{2}\left(\varepsilon_{2}\right)\right) .
$$

Since $s_{1}^{2}\left(\varepsilon_{1}\right)=X_{1} X_{2} \varepsilon_{1}$, and $s_{1}^{2}\left(\varepsilon_{2}\right)=\left(1-X_{1}\right) \varepsilon_{1}+\varepsilon_{2}$, the invariance of $h$ and the chosen value of $h\left(\varepsilon_{1}, \varepsilon_{1}\right)$ imply

$$
h\left(\varepsilon_{1}, \varepsilon_{2}\right)=\frac{-X_{2}}{1-X_{2}}, \quad h\left(\varepsilon_{2}, \varepsilon_{2}\right)=\frac{1-X_{2} X_{3}}{\left(1-X_{2}\right)\left(1-X_{3}\right)} .
$$

We can proceed in a like manner. Thus the $n \times n$-matrix $h(X)=\left(h_{i j}\right)$ of the skew hermitian form is

$h(X)=\left(\begin{array}{cccccc}\frac{1-X_{1} X_{2}}{\left(1-X_{1}\right)\left(1-X_{2}\right)} & -\frac{1}{1-X_{2}} & 0 & 0 & \ldots & 0 \\ -\frac{X_{2}}{1-X_{2}} & \frac{1-X_{2} X_{3}}{\left(1-X_{2}\right)\left(1-X_{3}\right)} & -\frac{1}{1-X_{3}} & 0 & \ldots & 0 \\ 0 & -\frac{X_{3}}{1-X_{3}} & \frac{1-X_{3} X_{4}}{\left(1-X_{3}\right)\left(1-X_{4}\right)} & -\frac{1}{1-X_{4}} & 0 & \ldots \\ \cdots & \cdots & \cdots & \cdots & \ldots & \end{array}\right)$.

Thus the invariance of $h$ implies that $h$ is the above matrix (once the value of $h\left(\varepsilon_{1}, \varepsilon_{1}\right)$ is normalised as above).

The natural formula for $h(X)$ given above takes values in the ring $R^{\prime}$ which is generated by $R$ together with the inverse of the element $\prod_{i=1}^{n+1}\left(1-X_{i}\right)$. One can clear denominators and ensure that $h(X)$ takes values in $R$. Therefore $g_{n}(X)\left(P_{n+1}\right) \subset U(h(X))(S)$, where $S$ is the sub-ring of $R$ invariant under the involution $f \mapsto \bar{f}$ on $R$ given by $X_{i} \mapsto X_{i}^{-1}$. The unitary group $U(h)$ is an affine algebraic group scheme defined over $S$ :

$$
U(h)(S)=\left\{g \in G L_{n}(R): h(g v, g w)=h(v, w) \forall v, w \in R^{n}\right\} .
$$


The following result is due to [2] (the skew hermitian form in [2] is for a different basis) :

Lemma 15. [1] The skew hermitian form on $\left(R^{n}\right)$ defined by the matrix $h(X)$ is invariant under the action of the pure braid group. Therefore, $\rho_{X}\left(P_{n+1}\right) \subset U(h)(S)$, where $S \subset R$ is the sub-ring of elements invariant under the involution.

[2] The matrix $h(X)$ has determinant

$$
\frac{1-X_{1} X_{2} \cdots X_{n} X_{n+1}}{\left(1-X_{1}\right)\left(1-X_{2}\right) \cdots\left(1-X_{n}\right)\left(1-X_{n+1}\right)} .
$$

[3] In particular, the skew Hermitian form $h$ is non-degenerate.

Proof. The form $h$ was constructed under the assumption that it was invariant under $P_{n+1}$. Hence we need only prove part [2] of the lemma. Part [2] of the lemma is proved by induction. Put $X=\left(X_{1}, X^{\prime}\right)$ where $X^{\prime}$ is the $n$-tuple $\left(X_{2}, \cdots, X_{n+1}\right)$; write $X^{\prime}=\left(X_{2}, X^{\prime \prime}\right)$ where $X^{\prime \prime}$ is the $n$-1-tuple $\left(X_{3}, X_{4}, \cdots, X_{n+1}\right)$. Expand the determinant of the $n \times n$ matrix $h_{n}(X)=h(X)$ by the first row; then $h_{n}(X)$ may be expressed in terms of $h_{n-1}\left(X^{\prime}\right)$ and $h_{n-2}\left(X^{\prime \prime}\right)$ :

$$
h_{n}(X)=\frac{1-X_{1} X_{2}}{\left(1-X_{1}\right)\left(1-X_{2}\right)} h_{n-1}\left(X^{\prime}\right)-\frac{X_{2}}{\left(1-X_{2}\right)^{2}} h_{n-2}\left(X^{\prime \prime}\right) .
$$

Now induction on $n$ implies the formula for the determinant of $h_{n}(X)$.

Suppose that $\mathfrak{a} \subset R$ is a non-zero ideal invariant under the involution $f \mapsto \bar{f}$ on $R$. Then the quotient map $R \rightarrow R / \mathfrak{a}$ induces a homomorphism $G L_{n}(R) \rightarrow G L_{n}(R / \mathfrak{a})$; the skew hermitian form $h$ descends to a skew hermitian form $h_{\mathfrak{a}}$ on the quotient module $(R / \mathfrak{a})^{n}$ and hence we have a homomorphism $U(h)(S) \rightarrow U\left(h_{\mathfrak{a}}\right)(S / \mathfrak{a} \cap S)$; therefore we have a representation $g_{n, \mathfrak{a}}: P_{n+1} \rightarrow U\left(h_{\mathfrak{a}}\right)(S / \mathfrak{a} \cap S)$.

Now consider a homomorphism from the ring $R=\mathbb{Z}\left[X_{i}^{ \pm 1} ; 1 \leq i \leq\right.$ $n+1]$ into the ring $\mathbb{Z}\left[\omega_{d}\right]$ of integers in the cyclotomic extension $E_{d}=$ $\mathbb{Q}\left(e^{\frac{2 \pi i}{d}}\right)=\mathbb{Q}\left(\omega_{d}\right)\left(\omega_{d}\right.$ is the primitive $d$-th root of unity $\left.e^{\frac{2 \pi i}{d}}\right)$. This homomorphism is given by $X_{i} \mapsto t_{i}$ where $t_{i}=\omega_{d}^{k_{i}}$ is a $d$-th root of unity. Since all the $k_{i}$ are co-prime to $d$, it follows that the group generated by each of the $t_{i}$ is all of $\mathbb{Z} / d \mathbb{Z}$, the group of $d$-th root of unity. Under the homomorphism $R \rightarrow \mathbb{Z}\left[\omega_{d}\right]$, the sub-ring $S$ maps into the ring $O_{d}$ of integers in the totally real sub-field $\mathbb{Q}\left(2 \cos \frac{2 \pi}{d}\right)$ of the cyclotomic field $E_{d}$. Hence we have the composite representation, 
denoted

$$
g_{n}(k, d): P_{n+1} \rightarrow U(h)(S) \rightarrow U(h)\left(O_{d}\right),
$$

where $k$ is the $(n+1)$-tuple of integers $\left(k_{1}, k_{2}, \cdots, k_{n+1}\right)$.

The following is the main result of the paper, from which Theorem 1 will be deduced.

Theorem 16. Suppose $d \geq 3, n \geq 2 d$ and all the integers $k_{i}$ are coprime to $d$. Then the image $\Gamma_{n}=\Gamma=g_{n}(k, d)\left(P_{n+1}\right)$ (of the Gassner representation $g_{n}(k, d)$ at primitive $d$-th roots of unity) is a subgroup of finite index in the integral unitary group $U(h)\left(O_{d}\right)$. In other words, the "monodromy group" $g_{n}(k, d)\left(P_{n+1}\right)$ is an arithmetic group.

The full braid group $B_{n+1}$ has a representation, called the reduced Burau representation ([6], p.118, Example 3),

$$
\rho_{n}(q): B_{n+1} \rightarrow G L_{n}\left(\mathbb{Z}\left[q, q^{-1}\right]\right)
$$

Since the restriction to $P_{n+1}$ of the reduced Burau representation at primitive $d$-th roots of unity is the reduced Gassner representation $g_{n}(k, d)$ evaluated at primitive $d$-th roots of unity (when all the $k_{i}$ are equal to 1), the following Theorem is a special case of Theorem 16.

Theorem 17. If $d \geq 3$ and $n \geq 2 d$ and all the $k_{i}$ are 1 , then the image of the Burau representation

$$
\rho_{n}(d): B_{n+1} \rightarrow U(h)\left(O_{d}\right)
$$

evaluated at all primitive d-th roots of unity, is a subgroup of finite index. In other words, the monodromy group $\rho_{n}(d)\left(B_{n+1}\right)$ is an arithmetic group.

Theorem 17 was proved in [25], by using properties of the Burau representation at roots of unity, and by using induction for all $n \geq 2 d$. The proof of Theorem 16 is similar, and we use properties of the reduced Gassner representations at roots of unity. These properties are essentially well known, but we need a precise form of these results. Theorem 16 will be proved at the end of this section, after many preliminary results.

4.2. Irreducibility. As we have seen before, the reduced Gassner representation has a nondegenerate invariant skew hermitian form $h$ with values in the field of fractions $\Omega$ of the ring $R=\mathbb{Z}\left[X_{1}^{ \pm 1}, \cdots, X_{n+1}^{ \pm 1}\right]$ of Laurent polynomials, which is preserved by the group $P_{n+1}$ under the Gassner representation. This was determined on the basis $\varepsilon_{i}$ in subsection 4.1. By Lemma 14, it follows that the elements $s_{i}^{2}$ are complex reflections. 
Proposition 18. [1] If $R=\mathbb{Z}\left[X_{1}^{ \pm 1}, X_{2}^{ \pm 1}, \cdots, X_{n+1}^{ \pm 1}\right]$, and $\Omega$ is its quotient field, then the reduced Gassner representation

$$
G_{n}(X): P_{n+1} \rightarrow G L_{n}(R) \subset G L_{n}(\Omega),
$$

is irreducible.

[2] The central element $\Delta^{2} \in P_{n+1}$ where

$$
\Delta=\Delta_{n}=\left(s_{1} s_{2} \cdots s_{n}\right)\left(s_{1} s_{2} \cdots s_{n-1}\right) \cdots\left(s_{1} s_{2}\right)\left(s_{1}\right),
$$

acts by multiplication by the scalar $X_{1} X_{2} \cdots X_{n+1}$ on the reduced Gassner representation.

[3] If $W \subset R^{n}$ is an additive subgroup stable under the action of $P_{n+1}$ then there exists a scalar $\lambda \in R$ such that $\lambda\left(R^{n}\right) \subset W$.

Proof. [1] At the beginning of this subsection, we have verified that the conditions of Lemma 7 are satisfied. By the result of [12] quoted earlier, $\Omega^{n}$ admits a nondegenerate hermitian form. Therefore, by Lemma 7. the representation $g_{n}(X)$ is absolutely irreducible.

[2] The element $\Delta^{2}$ is central in $P_{n+1}$; by part [1], the central element acts by a scalar, call it $\lambda$. Write $\pi_{n+1}$ for the product $X_{1} X_{2} \cdots X_{n+1}$. We compute the scalar $\lambda$ by finding the effect of $\Delta^{2}$ on the element $e_{1}$. Consider the element $x_{1} \in F_{n+1}$. A calculation (see the formulae for the action of $s_{i}$ on the free group $F_{n+1}$ in subsection (3.2)), shows that the action of $\Delta^{2}$ on $x_{1}$ is given by (notation: in a group, ${ }^{y}(x)=y x y^{-1}$ )

$$
\Delta^{2}\left(x_{1}\right)={ }^{x_{1} x_{2} \cdots x_{n+1}}\left(x_{1}\right) \text {. }
$$

In the semi-direct product $K^{a b} \rtimes F_{n+1}$, the element $x_{i}$ maps to $\bar{x}_{i}=$ $\left(e_{i}, X_{i}\right)=e_{i} X_{i}$. Hence this equation then becomes

$$
\begin{aligned}
\Delta^{2}\left(e_{i}, X_{i}\right) & =\Delta^{2}\left(e_{1} X_{1}\right)=\left(v \pi_{n+1}\right)\left(e_{1} X_{1}\right) \pi_{n+1}^{-1} v^{-1}= \\
& =\left(\pi_{n+1} e_{1}+\left(1-X_{1}\right) v, X_{1}\right)
\end{aligned}
$$

Comparing the vector parts, we get

$$
\Delta^{2}\left(e_{1}\right)=X_{1} X_{2} \cdots X_{n+1}\left(e_{1}\right)+\left(1-X_{1}\right) v .
$$

This is in the Gassner representation space $K^{a b}$. Going modulo the line through $v$, we see that

$$
\Delta^{2}\left(e_{1}\right)=\pi_{n+1} e_{1}=X_{1} X_{2} \cdots X_{n+1} e_{1} \quad(\bmod R v)
$$

in the reduced Gassner representation. This proves part [2] of the Proposition. 
We will now prove part [3]. Fix $i$ with $1 \leq i \leq n$. The group $P_{i+1}$ operates on the $R$ module generated by $\varepsilon_{1}, \cdots, \varepsilon_{i}$. This module is nothing but the reduced Gassner representation $g_{i}(X)$. Consequently, the element $C_{i}=\left(\Delta_{i}^{2}\right)$ acts by the scalar $X_{1} \cdots X_{i+1}$ on the vectors $\varepsilon_{1}, \cdots, \varepsilon_{i}$. Moreover, $s_{i}^{2}\left(\varepsilon_{i}\right)=X_{i} X_{i+1}\left(\varepsilon_{i}\right)$. In particular, the group $H_{i}$ generated by the elements $X_{1} \cdots X_{i+1}, X_{1} \cdots X_{i+2}, \cdots, X_{1} \cdots X_{n+1} ; X_{i} X_{i+1}$ has the property that the $\Gamma$ module generated by $\varepsilon_{i}$ contains all elements of the form $h\left(\varepsilon_{i}\right)$ for every $h \in H_{i}$. Note that $H_{i}$ is also the group generated by the elements $X_{1} \cdots X_{i+1}, X_{i+2}, \cdots, X_{n+1}, X_{i} X_{i+1}$ (successive ratios of the previous set of elements, together with the first and the last one of the previous set of elements).

Thus the group $H$ generated by $H_{1}, \cdots, H_{n}$ is the group generated by $X_{1} X_{2}, X_{3}, \cdots, X_{n+1}$ (contribution from $i=1$ ) and $X_{2} X_{3}$ (contribution from $i=2$ ). This is clearly the group generated by $X_{1}, \cdots, X_{n+1}$. By Corollary 2, this means that there exists a $\lambda \neq 0$ in $R$ such that $\lambda X_{1}^{\mathbb{Z}} \cdots X_{n+1}^{\mathbb{Z}}\left(\varepsilon_{i}\right)$ lies in the $\Gamma$-module generated by all the $\varepsilon_{i}$. Since monomials in the $X_{i}$ generate $R$, this means that $\lambda\left(R \varepsilon_{j}\right) \subset \mathbb{Z}[\Gamma] \varepsilon_{i}$ for all $i, j$.

Now, the additive group $W$ stable under the action of $\Gamma=P_{n+1}$ has the property that it contains a non-zero scalar multiple $\mu\left(\varepsilon_{i}\right)$ for all $i$ by Lemma 7. Hence $\lambda \mu\left(R \varepsilon_{i}\right) \subset W$. This is [3] of the proposition.

Notation. Suppose $\mathfrak{a}$ is a prime ideal in $R$ invariant under the involution on $R$ given by $X_{i} \mapsto X_{i}^{-1}$ for each $i$. Consider the integral domain $A=R / \mathfrak{a}$. Let $B$ denote the invariants in $A$ under the involution. We get the corresponding reduced Gassner representation $g_{n}(A)$ on the module $\oplus_{i=1}^{n} A \varepsilon_{i}$ of $P_{n+1}$. Write $t_{i}$ for the image of $X_{i}$ under the quotient map $R \rightarrow A=R / \mathfrak{a}$. The skew hermitian form $h$ reduced modulo $\mathfrak{a}$ gives a skew hermitian form on $A^{n}$ and the image of $P_{n+1}$ under $g_{n}(A)$ lies in $U(h)(A)$. Let $E$ denote the quotient field of $A$. Then $W(A)=\oplus A \varepsilon_{i}$ is a subgroup of the $E$-vector space $W(E)=\oplus E \varepsilon_{i}$.

Proposition 19. [1] If $t_{1} \cdots t_{n+1} \neq 1$ then $h(A)$ is non-degenerate and the representation $g_{n}(A)$ is irreducible.

[2] The central element $\Delta_{n}^{2}$ of $P_{n+1}\left[\right.$ where $\left.\Delta_{n}=\left(s_{1} \cdots s_{n}\right) \cdots\left(s_{1} s_{2}\right) s_{1}\right]$ acts by the scalar $t_{1} \cdots t_{n+1}$ on the representation $g_{n}(A)$.

[3] If $t_{1} \cdots t_{n+1} \neq 1$ then every nonzero $P_{n+1}$-invariant subgroup of $A^{n}=\oplus A \varepsilon_{i}$ contains $\lambda\left(A^{n}\right)$ for some non-zero element $\lambda \in A$. 
The proof of Proposition 18 can be repeated for the quotient $R / \mathfrak{a}$ in place of $R$.

\section{Proof of Theorem 16}

We will first prove some preliminary results on the reduced Gassner representation at $d$-th roots of unity. We will then use induction to deduce Theorem 16 from these results.

5.1. The reduced Gassner representation at roots of unity. Consider the reduced Gassner representation $g_{n}(k): P_{n+1} \rightarrow G L_{n}(R)$ where $R$ s the Laurent polynomial ring in $n+1$ variables $X_{i}$ with integral coefficients and $R^{n}$ is the free module $W_{n}(X)=\oplus_{i=1}^{n} R \varepsilon_{i}$. We now specialise to $X_{i} \mapsto t_{i}=t^{k_{i}}$. The resulting representation is from $P_{n+1}$ into $G L_{n}\left(A_{d}\right) \subset G L_{n}\left(E_{d}\right)$ where $E_{d}$ is the $d$-th cyclotomic extension of $\mathbb{Q}$ and $A_{d}$ the ring of integers in $E_{d}$, and is denoted $g_{n}(k, d)$. Denote the vector space $W_{n}(k, d)$.

Lemma 20. The reduced Gassner representation evaluated at all primitive d-th roots of unity is irreducible if and only if $t_{1} t_{2} \cdots t_{n+1} \neq 1$.

When $t_{1} \cdots t_{n+1}=1$, the representation space $W_{n}(k, d)$ of the representation $g_{n}(k, d)$ contains a non-zero invariant vector $w$ and the restriction of the quotient representation $W_{n}(k, d) / E_{d} w$ to the subgroup $P_{n}$ is isomorphic to $g_{n-1}(k, d)$. If we denote the quotient representation by $\bar{g}_{n}(k, d)$, then $\bar{g}_{n}(k, d)$ is irreducible.

If we denote by $\pi_{i}$ the product $t_{1} t_{2} \cdots t_{i}$, then the invariant element $w \in W_{n}(k, d)$ is given by

$$
w=\sum\left(1-\pi_{i}\right) \varepsilon_{i}
$$

Proof. If $t_{1} \cdots t_{n+1} \neq 1$, then the specialisation of the Hermitian form $h$ at $t_{1}, \cdots, t_{n+1}$ has non-zero determinant, by Lemma 15. Therefore, by Lemma 7 , the representation $g_{n}(k, d)$ is irreducible.

Suppose $t_{1} t_{2} \cdots t_{n+1}=1$. Consider the element

$$
v=e_{1}+t_{1} e_{2}+\cdots+t_{1} t_{2} \cdots t_{n-1} e_{n}+t_{1} t_{2} \cdots t_{n+1} e_{n+1} .
$$

This is the vector part of the $P_{n+1}$ invariant element $x_{1} x_{2} \cdots x_{n+1}$ in the semi-direct product $K^{a b} \rtimes t^{\mathbb{Z}}$, and is hence invariant. The expression for $v$ in terms of $\varepsilon_{i}$ and $v_{n+1}$ shows that

$$
v=\sum_{i=1}^{n}\left(1-t_{1} t_{2} \cdots t_{i}\right) \varepsilon_{i}+\left(1-t_{1} \cdots t_{n+1}\right) v_{n+1} .
$$


By assumption on the $t_{i}$, the coefficient of $v_{n+1}$ is zero, and hence $v$ lies in the reduced Gassner representation $W_{n}(k, d)$ (the span of the $\varepsilon_{i}$ ).

Let $P_{n}$ be the set of pure braids in the group generated by $s_{2}, s_{3}, \cdots, s_{n}$. The module $W=W_{n}(k, d)$ is spanned by the vector $v$ and $\varepsilon_{2}, \cdots, \varepsilon_{n}$. Therefore, $g_{n}(k, d)$ restricted to $P_{n}$ splits into a direct sum of the modules $R v$ and $g_{n-1}(k, d)$; the latter is irreducible by part [1], since $t_{2} t_{3} \cdots t_{p+1}=t_{1}^{-1} \neq 1$. We have therefore proved that the representation $\overline{g_{n}(k, d)}$ restricted to $P_{n}$ is the representation $g_{n-1}(k, d)$ and is irreducible for the subgroup $P_{n}$; hence it is irreducible for the bigger group $P_{n+1}$.

The lemma is due essentially to Abdulrahim [1] and [2]. We have derived the lemma since we need the explicit formula for the invariant element, in terms of the basis $\varepsilon_{i}$.

\subsection{Gassner representations with degenerate Hermitian forms.} Suppose that $t_{i}$ are all primitive $d$-th roots of unity and that $t_{1} t_{2} \cdots t_{p}=$ 1. Consider the basis $\varepsilon_{1}, \cdots, \varepsilon_{p-1}$ of $W$. This contains the element

$$
w=\sum_{i=1}^{p-1}\left(1-t_{i}\right) \varepsilon_{i} .
$$

Therefore, it is part of a basis of $W: w, \varepsilon_{2}, \cdots, \varepsilon_{p-1}$. Since $w$ is invariant, with respect to this basis, every element $g$ of the pure braid group $P_{p}$ has the matrix form

$$
\left(\begin{array}{cc}
1 & v \\
0_{p-2} & \alpha
\end{array}\right)
$$

where $\alpha$ is the matrix of $g$ acting on the quotient $W / E_{d} w$ with respect to the basis $\varepsilon_{2}, \varepsilon_{3}, \cdots, \varepsilon_{p-1}$. The element $C^{\prime}=\left(\Delta^{\prime}\right)^{2}$ where

$$
\Delta^{\prime}=\left(s_{2} \cdots s_{p}\right)\left(s_{2} \cdots s_{p-2}\right) \cdots\left(s_{2} s_{1}\right)\left(s_{2}\right),
$$

is central in the pure braid group $P_{p-2}$ and hence acts by a scalar on the irreducible representation; the scalar is $t_{2} t_{3} \cdots t_{p}=t_{1}^{-1} \neq 1$ (by part [2] of Proposition 19). Therefore, the commutator

$$
u=\left[g,\left(\Delta^{\prime}\right)^{2}\right]=\left(\begin{array}{cc}
1 & v^{\prime} \\
0_{p-2} & 1_{p-2}
\end{array}\right)
$$

acts by an upper triangular unitary matrix.

Proposition 21. Let $t_{1}, \cdots, t_{p}$ be primitive $d$-th roots of unity with $t_{1} \cdots t_{p}=1$. Consider the representation $g(p, t): P_{p} \rightarrow U(h)\left(O_{d}\right)$, the 
reduced Gassner representation $g(p, X)$ specialised to $X_{i} \mapsto t_{i}$. Then

[1] The unitary group $U(h)$ has a unipotent radical isomorphic to the $P_{p-1}$ module $W^{*}$, where $W$ is the reduced Gassner representation $g(p-1, t)$.

[2] If $g=s_{1}^{2}$ and $\Delta^{\prime}$ is as in the preceding paragraph, then the image of the element $u=\left[g,\left(\Delta^{\prime}\right)^{2}\right]$ under the reduced Gassner representation $g(p, t)$ is not identity.

[3] The conjugates $\left\{h u h^{-1}\right\}$ of $u$ for $h \in P_{p-1}$ generate a subgroup of finite index in the integral additive subgroup $W^{*}\left(O_{d}\right)$ of $W^{*}$.

Proof. Part [1] is obvious.

The vector

$$
w=\sum_{i=1}^{p}\left(1-t_{1} \cdots t_{i}\right) \varepsilon_{i}
$$

(notice that the coefficient of $\varepsilon_{p}$ is zero) is orthogonal to all the vectors $\varepsilon_{1}, \cdots, \varepsilon_{p}$ and is invariant under all of $P_{p}$. We can consider the basis $B^{\prime}$ given by $w, \varepsilon_{2}, \cdots, \varepsilon_{p}$ of the vector space $W=\oplus_{i=1}^{p} \varepsilon_{i}$. Now $s_{1}^{2}$ fixes $w$. By Lemma 14, we have the equalities $s_{1}^{2}\left(\varepsilon_{2}\right)=\varepsilon_{2}+\left(1-t_{1}\right) \varepsilon_{1}=$ $\varepsilon_{2}+w-\left(1-t_{1} t_{2}\right) \varepsilon_{2}-\cdots-\left(1-t_{1} \cdots t_{p}\right) \varepsilon_{p}$, and $s_{1}^{2}\left(\varepsilon_{i}\right)=1$ for $i \geq 3$. Therefore the matrix of $s_{1}^{2}$ in this basis $B^{\prime}$ is of the form

$$
g(p, t)\left(s_{1}^{2}\right)=\left(\begin{array}{ccccc}
1 & 1 & 0 & \ldots & 0 \\
0 & t_{1} t_{2} & 0 & \ldots & 0 \\
\ldots & \ldots & \ldots & \ldots & \ldots \\
0 & \ldots & 0 & 0 & 1
\end{array}\right)
$$

Consider the pure braid group $P_{p-1}^{\prime}$ generated by $s_{2}^{2}, \cdots, s_{p}^{2}$. The subspace spanned $W^{\prime}$ by $\varepsilon_{2}, \cdots, \varepsilon_{p}$ is left stable under $P_{p-1}^{\prime}$ and the resulting representation is the Gassner representation for $P_{p-1}^{\prime}$. Consequently, the element $\left(\Delta^{\prime}\right)^{2}$ acts by the scalar $c=t_{2} \cdots t_{p}=t_{1}^{-1} \neq 1$ on $W^{\prime}$. Moreover, $\left(\Delta^{\prime}\right)^{2}$ fixed the vector $w$. Hence with respect to the basis $B^{\prime}$ the element $\left(\Delta^{\prime}\right)^{2}$ has the matrix

$$
g(p, t)\left(\left(\Delta^{\prime}\right)^{2}\right)=\left(\begin{array}{ccccc}
1 & 0 & 0 & \ldots & 0 \\
0 & c & 0 & \ldots & 0 \\
\ldots & \ldots & \ldots & \ldots & \ldots \\
0 & \ldots & 0 & 0 & c
\end{array}\right)
$$


It is then clear from the above matrix forms that the commutator of $s_{1}^{2}$ and $\left(\Delta^{\prime}\right)^{2}$ has the matrix form

$$
g(p, t)(u)=g(p, t)\left(\left[s_{1}^{2},\left(\Delta^{\prime}\right)^{2}\right]\right)=\left(\begin{array}{cccc}
1 & t_{1}^{-1} t_{2}^{-1}\left(1-c^{-1}\right) & \ldots & 0 \\
0 & 1 & \cdots & 0 \\
\cdots & \cdots & \ldots & \ldots \\
0 & \ldots & 0 & 1
\end{array}\right) .
$$

In other words, this lies in the vector group $W^{*}\left(O_{d}\right)$ and is a nonidentity element (since $c \neq 1$ ). This proves part [2].

Consider the image (under $g(p, t)$ ) of the group generated by the conjugates $h u h^{-1}$ of $u$ by elements $h$ (of the smaller braid group $P_{p-1}$ generated by $\left.s_{2}, s_{3}, \cdots, s_{p-1}\right)$. This image may be identified with an additive subgroup $A$ of the vector group $W^{\prime *}$ where $W^{\prime *}$ is dual of the reduced Gassner representation $W^{\prime}$ at $d$-th roots of unity for $P_{p-1}$. It follows from part [3] of Proposition 19 that the additive subgroup $A$ contains a subgroup of finite index in the (dual of the) vector group

$$
W^{\prime}\left(A_{d}\right) \simeq \oplus_{i=2}^{p} A_{d} \varepsilon_{i}
$$

We now consider the "next" pure braid group $P_{p+1}$, with $t_{1} t_{2} \cdots t_{p}=$ 1. The Gassner representation takes $P_{p+1}$ into a unitary group $U(h)$. The product $t_{1} t_{2} \cdots t_{p+1}=t_{p+1} \neq 1$ since all the $t_{i}$ are primitive $d$ th roots of unity. With respect to the basis $w, \varepsilon_{2}, \cdots \varepsilon_{p-1}$ and $\varepsilon_{p}$, the matrix of the commutator $u=\left[s_{1}^{2},\left(\Delta^{\prime}\right)^{2}\right]$ now takes the form

$$
u=\left(\begin{array}{ccc}
1 & v & \lambda \\
0_{p-2} & 1_{p-2} & \bar{v} \\
0 & 0 & 1
\end{array}\right)
$$

and the group generated by the conjugates $\left\{m u m^{-1}: m \in P_{p-1}\right\}$ is a subgroup of finite index in the Heisenberg group $H(X)$ where $H(X)$ is the subgroup of $U(h)$ which acts unipotently on the flag

$$
E_{d} w \subset E_{d} w+X \subset V,
$$

where $X$ is the span of $\varepsilon_{2} \cdots, \varepsilon_{p-1}$, and $V$ is the span of $\varepsilon_{1}, \cdots, \varepsilon_{p}$ (the reduced Gassner representation). Hence the Gassner image contains a subgroup of finite index in the unipotent integral Heisenberg group $H(X)\left(O_{d}\right)$.

Proposition 22. Let $t_{1}, t_{2}, \cdots, t_{p}, t_{p+1}$ be primitive $d$-th roots of unity such that $t_{1} t_{2} \cdots t_{p}=1$. Denote by $\Gamma_{p}$ the image of the Gassner representation

$$
g_{p}(k, d): P_{p+1} \rightarrow U(h)\left(O_{d}\right) \subset G L_{p}\left(A_{d}\right)
$$


Then

[1] there exist two opposite maximal parabolic subgroups $P$ and $P^{-}$ of $U(h)$ such that the image $\Gamma_{p}$ intersects the integral unipotent radicals $U_{P}\left(O_{d}\right)$ and $U_{P}^{-}\left(O_{d}\right)$ in subgroups of finite index.

[2] In particular, if $K-\operatorname{rank}(U(h)) \geq 2$, then the image $\Gamma_{p}$ is an arithmetic subgroup of (i.e. subgroup of finite index in) $U(h)\left(O_{d}\right)$.

Proof. Since $t_{1} t_{2} \cdots t_{p+1}=t_{p+1} \neq 1$, it follows that the hermitian form $h=h_{p}$ is non-degenerate. Therefore, the unitary group $U(h)$ is reductive. Denote by $V=V_{p}=E_{d}^{p}$ the natural representation of $U(h)\left(E_{d}\right.$ is the $d$-th cyclotomic extension of $\mathbb{Q}$ ).

We have already seen in the paragraph preceding the statement of the proposition, that $\Gamma_{p}$ contains a subgroup $U_{0}$ of finite index in the integer points of the Heisenberg group $H(X)$. Recall that $H(X)$ is the unipotent radical of a parabolic subgroup $P$ (i.e. $P$ is the normaliser of $H(X)$ in the unitary group $U(h))$. We will now prove that there exists a conjugate of $U_{0}$ in $\Gamma_{p}$ which is an arithmetic subgroup of an opposite unipotent radical.

By assumption, there exists an isotropic vector $v=\sum_{i=1}^{p-1}\left(1-\pi_{i}\right) \varepsilon_{i}$ (it is orthogonal to all the $\varepsilon_{i}$ with $i \leq p-1$ and is therefore orthogonal to itself). Consequently, we may find a basis

$$
v=w_{1}, \cdots, w_{r}, x_{1}, \cdots, x_{s}, w_{1}^{*}, \cdots, w_{r}^{*}
$$

of $V$ where $x_{i}$ are orthogonal to the $w_{i}$ and $w_{i}^{*}, w_{i}$ are isotropic mutually orthogonal vectors, similarly, $w_{j}^{*}$ are mutually orthogonal isotropic vectors, and $w_{j}^{*}\left(w_{i}\right)=\delta_{i j}$ (the Kronecker delta symbol). The intersection of the diagonals with $U(h)$ then gives a maximal torus defined over $K$, which is maximally $K$-split over $K . N(T)$ denotes the $K$-Weyl group and $\kappa$ denotes the longest element in the $K$-Weyl group.

The following Zariski density statement is very likely true in greater generality (cf. Lemma (11.5) of [8] and Lemma (4.4) of [13] ), but we will need only this weaker version in the course of the proof.

Lemma 23. Suppose that $\Gamma \subset U(h)\left(O_{d}\right)$ is the image of the pure braid group under the reduced Gassner representation. Suppose that $\Gamma_{n}$ contains a finite index subgroup of the Heisenberg group which can be viewed as the integral unipotent radical of a (maximal) parabolic $K_{d^{-}}$ subgroup of $U(h)$ and that $\Gamma$ acts irreducibly on the reduced Gassner 
representation (i.e. suppose that $t_{1} t_{2} \cdots t_{n+1} \neq 1$ ). Then, the Zariski closure of $\Gamma_{n}$ contains the special unitary group $S U(h)$.

Proof. We will use Proposition 8 of section 2, The irreducibility of the action of $\Gamma_{n}$ implies that the Zariski closure (intersected with $S L_{n}(\mathbb{C})$ ) is reductive. Since $\Gamma_{n}$ is assumed to contain a finite index subgroup of the group of integral points of the unipotent radical given by the Heisenberg group, it follows that if $H$ is the Zariski closure of $\Gamma_{n}$ then $H$ contains $U$, the group of the form in Proposition 8. Then Proposition 8 implies the Lemma.

We continue with the proof of Proposition 22, By Lemma 23, it follows that $\Gamma_{p}$ intersects the big Bruhat cell (the Zariski open set $U \kappa P$ where $\kappa$ is the longest Weyl group element above). Let $\gamma$ lie in the image $\Gamma_{p}$ and also in the big Bruhat cell. Then $\gamma P \gamma^{-1}=P^{\prime}$ is opposite to $P$ and hence $\gamma U_{0} \gamma^{-1}$ lies in $\Gamma_{p}$. Therefore, the first part of the proposition follows.

To prove the second part, note that $U(h)\left(O_{d}\right)$ is a higher rank lattice containing $\Gamma$. By part 1 of the proposition, $\Gamma$ contains a finite-index subgroup of integral points of the unipotent radical of a parabolic $K$ subgroup. By Lemma 23, $\Gamma$ is Zariski dense. Hence by Theorem 4, the second part follows.

5.3. Proof of Theorem 16. Recall the notation: let $k_{1}, k_{2}, \cdots, k_{n+1}$ be integers with $1 \leq k_{i} \leq d-1$ and co-prime to $d$. Let $\omega_{d}=e^{2 \pi i / d}$ be a primitive $d$-th root of unity; write $t_{i}=\omega_{d}^{k_{i}}$. Let $\mathbb{Q}\left(\omega_{d}\right)$ be the $d$-th cyclotomic extension and $A_{d}$ the ring of integers in $E_{d}$; denote by $K_{d}$ the maximal totally real sub-field $\mathbb{Q}(2 \cos (2 \pi / d))$ of $E_{d}$ and $O_{d}$ the ring of integers in $K_{d}$. Denote by $g_{n}(k, d): P_{n+1} \rightarrow U(h)\left(O_{d}\right) \subset G L_{n}\left(A_{d}\right)$ the reduced Gassner representation specialised at $X_{i} \mapsto t_{i}$. In this section, for ease of notation (since we use induction and have to deal with many indices) we denote by $V_{n}$ the $E_{d}$ vector space spanned by $\varepsilon_{1}, \cdots, \varepsilon_{n}$ (this is the same as $W_{n}(k, d)$ ).

Suppose $s \in B_{n+1}$ is an element of the full braid group, whose image in $S_{n+1}$ is the permutation $\sigma$. Now $\sigma$ operates on the Laurent polynomial ring $\mathbb{Z}\left[X_{1}^{ \pm 1}, \cdots X_{n+1}^{ \pm 1}\right]$ by permutations of the $X_{i}$ 's. Given $s$, denote by $u$ the $n \times n$ matrix $\left(u_{i j}\right)$ such that

$$
\sigma\left(\varepsilon_{i}\right)=\sum_{j=1}^{n} u_{j i} \varepsilon_{j} .
$$


Recall that $B_{n+1}$ operates on the Gassner module $W \otimes \Omega$ by automorphisms of the abelian group, but not linearly over $\Omega$. Let $s \in B_{n+1}, \sigma$ its image in $S_{n+1}$. It easily follows from the construction of the Gassner representation that, for all $g$ in the pure braid group $P_{n+1}$, we have the equality

$$
g(n, X)\left(s g s^{-1}\right)=\sigma(u) \sigma\left(g(n, X)(g) \sigma(u)^{-1} .\right.
$$

In particular, the image of $g(n, X)$ is a conjugate, by an element in $G L_{n}(R)$, of the image of the twisted representation $\sigma(g(n, X))=$ $g(n, \sigma(X))$. Therefore, if we wish to prove that the specialisation of $g(n, X)$ at some roots of unity is arithmetic, it is enough to prove it for the "twisted" representation $\sigma(g(n, X))$ (the notation $\sigma(g(n, X))$ means that the matrix entries of $g(n, X)$ which are elements of $R$ acted upon by the permutation $\sigma$ of the variables $X_{i}$ ).

We now begin the proof of Theorem 16.

Proof. We first prove that there exist two elements $v, v^{\prime} \in E_{d}^{n}$ which are linearly independent and mutually orthogonal with respect to the hermitian form.

Consider the $d$ numbers $t_{1}, t_{1} t_{2}, \cdots, t_{1} t_{2} \cdots t_{d-1}$ and $t_{1} t_{2} \cdots t_{d}$. These are $d$ elements of the group $\mu_{d}$ of $d$-th roots of unity . Therefore, by the pigeon- hole principle, we have two possibilities. (a) One of these products is one, or (b) Two of them coincide.

Hence there exists a subset $I \subset\{1,2, \cdots, d-1, d\}$ consisting of $l$ consecutive integers such that $\prod_{i \in I} t_{i}=1$. Let $I=\{a+1, \cdots a+l\}$. Put

$$
v=\sum_{i=1}^{l-1}\left(1-t_{a+1} \cdots t_{a+i}\right) \varepsilon_{a+i} .
$$

By an earlier computation, $v$ is orthogonal to all the $\varepsilon_{a+i}$ with $1 \leq i \leq$ $a+l$. Note that in the expression of $v$ as a linear combination of the $\varepsilon_{i}$, the "last" basis vector $\varepsilon_{a+l}$ does not appear.

Similarly, there exists a subset $J \subset\{d+1, \cdots, 2 d\}$ consisting of $m$ consecutive numbers such that $\prod_{j \in J} t_{j}=1$; let $J=\{b+1, \cdots b+m\}$, where $b \geq l+1$. As before,

$$
v^{\prime}=\sum_{j=1}^{m-1}\left(1-t_{b+1} \cdots t_{b+j}\right) \varepsilon_{b+j},
$$


is isotropic and is orthogonal to all the $\varepsilon_{b+j}$ with $1 \leq j \leq m$. Since the indices of the $\varepsilon_{\mu}$ occurring in $v$ are of the form $\mu=a+i$ with $i \leq l-1$, it follows that $a+i \leq d-1$. Since the indices of $\varepsilon_{\nu}=\varepsilon_{b+j}$ occurring in the expression for $v^{\prime}$ are of the form $\nu=b+j \geq d+1$, it follows that $\nu-\mu \geq 2$. Therefore, $v, v^{\prime}$ are orthogonal.

Therefore the $E_{d}$-rank of the span of the vectors $\varepsilon_{a+1}, \cdots, \varepsilon_{a+l}$ and $\varepsilon_{b+1}, \cdots, \varepsilon_{b+m}$ is at least two.

By the remarks preceding the beginning of the proof of Theorem 16. we may assume that $a=0$ and $b=l$, after a permutation $\sigma$ of the indices, so that $t_{1} \cdots t_{l}=1$ and $t_{l+1} \cdots t_{l+m}=1$. Therefore $t_{1} \cdots t_{l+m+1}=t_{l+m+1} \neq 1$ and $V_{n}$ is non-degenerate if $n=l+m$. By Proposition 22, the group $\Gamma_{l+m}$ intersects two opposite integral unipotent radicals $U_{P}^{+}$and $U_{P}^{-}$in subgroups of finite index. By the conclusion of the preceding paragraph we get that for $n=l+m$, the group $U(h)$ has $K$-rank at least two, and therefore the group $\Gamma_{n}$ is arithmetic, by Theorem 4 .

If the Theorem is true for some $n \geq l+m$, then we will prove that it is true for $n+1$. There are several cases to consider.

Let $V=V_{n}$ (resp. $\left.V_{n+1}\right)$ be the span of $\varepsilon_{1}, \cdots, \varepsilon_{n}$ (resp. $\left.\varepsilon_{1}, \cdots, \varepsilon_{n+1}\right)$. Let $V_{n}^{\prime}$ be the span of $\varepsilon_{2}, \cdots, \varepsilon_{n+1}$. Then, the intersection $V_{n} \cap V_{n}^{\prime}$ is the span of $\varepsilon_{2}, \cdots, \varepsilon_{n}$. Therefore, $V_{n} \cap V_{n}^{\prime}$ contains a subspace $W^{\prime \prime}$ which is non-degenerate and contains an isotropic vector (e.g. take $W^{\prime \prime}$ to be the span of $\left.\varepsilon_{l}, \varepsilon_{l+1}, \cdots, \varepsilon_{l+m+1}\right)$.

Case 1. Assume that $V_{n}, V_{n}^{\prime}, V_{n+1}$ are all non-degenerate. Since $n \geq l+m$ the $K$-rank of $U\left(h_{n+1}\right)$ and $U\left(h_{n}\right)$ are both $\geq 2$. By induction assumption, $U\left(V_{n}\right) \cap \Gamma$ is arithmetic and $U\left(V_{n}^{\prime}\right) \cap \Gamma$ is arithmetic. By Lemma 5, $U\left(h_{n+1}\right)$ is also arithmetic.

Case 2. $V_{n+1}$ is non-degenerate but $V_{n}$ is degenerate. Then by Proposition 22, $U\left(h_{n+1}\right) \cap \Gamma$ is arithmetic. Similarly, if $V_{n+1}$ non-degenerate but $V_{n}^{\prime}$ is degenerate, $\Gamma_{n+1}$ can be proved to be arithmetic.

Case 3. $V_{n+1}$ is degenerate. Then $V_{n+1}$ contains a one dimensional null space $E_{d} v$ and by induction, the image of $\Gamma$ in $U\left(V_{n+1} / A v\right)$ is arithmetic. However, by part [3] of Proposition 21, the group $\Gamma$ intersects the integral unipotent radical of $U\left(V_{n+1}\right)$ in a finite index subgroup. Therefore, $\Gamma$ contains a finite index subgroup of the integral unipotent 
radical of $U(h)$ and maps onto a finite index subgroup of the reductive Levi part of $U(h)\left(O_{d}\right)$. Hence $\Gamma$ is a subgroup of finite index.

If $n \geq 2 d$, then in the above notation, $n \geq l+m$. Now the three induction steps proved above imply that the group $\Gamma_{n}$ is arithmetic.

\section{Homology of Cyclic Coverings}

In this section, we will view the first $\mathbb{Q}$-homology of certain index $d$ subgroups of the free group on $n+1$ generators, with essentially a direct sum of the reduced Gassner representation evaluated at $d$-th roots of unity. The proof is a little indirect, since it does not seem possible to get a natural basis of the homology of index $d$-subgroup, which generates the relevant Gassner representation. Instead, we replace the free group $F_{n+1}$ on $n+1$ genetators with a free product of $F_{n+1}$ and an auxilliary $\mathbb{Z}$ (the free product is then the free group on $n+2$ generators). It seems easier to identify the homology of a finite index subgroup of the latter, with a specialisation of the Gassner representation.

6.1. Image of homology. Let $k_{1}, k_{2}, \cdots, k_{n+1}$ be integers co-prime to $d$ with $1 \leq k_{i} \leq d-1$. Write $k=\left(k_{1}, k_{2}, \cdots k_{n+1}\right)$. We get a homomorphism from the free group $F_{n+1} \rightarrow \mathbb{Z} / d \mathbb{Z}$ (the latter written multiplicatively as $\left.q^{\mathbb{Z}} / q^{d \mathbb{Z}}\right)$ by sending $x_{i}$ to the element $q^{k_{i}}$. Denote by $K(k, d)$ the kernel to this map. We have thus an exact sequence

$$
1 \rightarrow K_{0}(k, d) \rightarrow F_{n+1} \rightarrow \mathbb{Z} / d \mathbb{Z} \rightarrow 1
$$

Being a subgroup of finite index (in fact of index $d$ ) in $F_{n+1}$, the group $K_{0}(k, d)$ is also free on $n_{0}^{\prime}$ generators, with

$$
\left(1-n_{0}^{\prime}\right)=d(1-(n+1)) \text {, i.e. } n_{0}^{\prime}=1+n d .
$$

Since the $k_{i}$ are co-prime to $d$, the image of $x_{i}$ generates $\mathbb{Z} / d \mathbb{Z}$; hence there exists an element $\xi \in F_{n+1}$ such that its image is $q$. Then the elements $z_{i}=x_{i} \xi^{-k_{i}}$ lie in the kernel $K_{0}(k, d)$; moreover, the elements $\xi$ and $\left\{z_{i}: 1 \leq i \leq n+1\right\}$ generate $F_{n+1}$. Hence the $z_{i}$ and $\xi^{d}$ generate the kernel $K_{0}(k, d)$ as a normal subgroup of $F_{n+1}$ : if we go modulo the normal subgroup generated by the $z_{i}$, then the resulting group is generated by the image of $\xi$ and maps onto $\mathbb{Z} / d \mathbb{Z}$.

The first homology group $V_{0}=K_{0}(k, d)^{a b} \otimes \mathbb{Q}$ with $\mathbb{Q}$ coefficients of $K_{0}(k, d)$ is therefore a vector space of dimension $n_{0}^{\prime}=1+n d$ over $\mathbb{Q}$. We have already seen that this homology group is a module over the quotient group $\mathbb{Z} / d \mathbb{Z}$, and is hence a module over the group ring $\mathbb{Q}[q] /\left(q^{d}-1\right)$. As a $\mathbb{Q}$ vector space, $V_{0}$ is generated by the elements 
$\xi^{j}\left(z_{i}\right):(2 \leq i \leq n+1$ and $0 \leq j \leq d-1)$ and the element $\xi^{d}$. Hence these elements form a basis of the first homology group over $\mathbb{Q}$.

We first find the invariants $V_{0}^{G}$ in $V_{0}$ under the action of the group $G=\mathbb{Z} / d \mathbb{Z}$. Since the image $q$ of $\xi$ generates $G$, it follows that $V_{0}=\left(V_{0} /\left(1+q+\cdots+q^{d-1}\right)\right) \oplus V_{0}^{G}$. In this decomposition, the element $q$ may be replaced by $q^{k_{i}}$ for any $i$ since $k_{i}$ is coprime to $d$.

We know that $\xi^{d} \in V_{0}$ is invariant. Moreover, $x_{i}^{d} \in V_{0}$ is invariant under conjugation by $x_{i}$; but the conjugation action of $F_{n+1}$ on $V_{0}$ descends to that of $G$ and each $x_{i}$ generates $G$. Therefore, $x_{i}^{d}$ is invariant under $G$. We write $x_{i}^{d}$ in terms of the $x_{j}=z_{i} \xi^{k_{i}}$ :

$$
x_{i}^{d}=z_{i}\left(1+q^{k_{i}}+\cdots+q^{k_{i}(d-1)}\right) .
$$

As an operator on $V_{0}$ multiplication by the element $M_{i}=1+q^{k_{i}}+\cdots+$ $q^{(d-1) k_{i}}$ is zero on non-invariants and $d$ on invariants. Therefore, $M_{i}$ is independent of $k_{i}$ and hence $x_{i}^{d}=z_{i}\left(1+q+\cdots+q^{d-1}\right)$. In other words the $\mathbb{Q}$-span of the $x_{i}^{d}$ is the space of all invariants $V_{0}^{G}$ :

$$
V_{0}^{G}=\sum_{i=1}^{n+1} \mathbb{Q}\left[x_{i}^{d}\right] .
$$

Consider the quotient $V_{0}^{n i}=V_{0} /\left(1+q+\cdots q^{d-1}\right) V_{0}$ (ni stands for non-invariants). This is a module over the quotient ring $\mathbb{Q}[q] /(1+$ $\left.q+\cdots q^{d-1}\right)$. By the discussion of the preceding paragraphs, the first homology $V_{0}$ is generated as a $\mathbb{Q}[q] /\left(q^{d}-1\right)$ module by (the images in the abelianisation of $K_{0}(k, d)$ of ) the elements $z_{i}$ and by $\xi^{d}$. Since $\xi$ commutes with $\xi^{d}$ it follows that in the homology group, $q\left(\xi^{d}\right)=\xi^{d}$; hence the augmentation ideal of the group ring $\mathbb{Q}[q] /\left(q^{d}-1\right)$ kills $\xi^{d}$. Therefore we see that the $z_{i}$ form a basis of the free module $V_{0}^{n i}$ as a module over the ring $R_{d}=\mathbb{Q}[q] /\left(1+q+\cdots q^{d-1}\right)$ :

$$
V_{0}^{n i}=R_{d}^{n} \text {. }
$$

We also have the free group on $n+2$ generators written $F_{n+1} * t^{\mathbb{Z}}$, with a natural inclusion of $F_{n+1}$ in $F_{n+2}$. On $F_{n+2}$ we have a homomorphism into $\mathbb{Z} / d \mathbb{Z}$ by sending $x_{i}$ to $q^{k_{i}}$ and $t$ to the standard generator $q$. Denote by $K(k, d)$ the kernel to this map; we have a short exact sequence

$$
1 \rightarrow K(k, d) \rightarrow F_{n+1} * t^{\mathbb{Z}} \rightarrow \mathbb{Z} / d \mathbb{Z} \rightarrow 1 .
$$

For similar reasons, $K(k, d)$ is free on $n^{\prime}$ generators where $n^{\prime}$ is given by the formula $n^{\prime}=1+(n+1) d$. Thus the first homology group $V=H_{1}(k, d)^{a b} \otimes \mathbb{Q}$ has dimension $(n+1) d+1$ over $\mathbb{Q}$ and is a module 
over the group ring $\mathbb{Q}[q] /\left(q^{d}-1\right)$. If we go modulo the action of the linear transformation $\left(1+q+\cdots+q^{d-1}\right)$ the resulting quotient of the homology group of $K(k, d)$ is denoted $V^{n i}$. Then, as before,

$$
V^{n i}=R_{d}^{n+1},
$$

is a free module over the ring $R_{d}$.

The natural inclusion of $F_{n+1}$ in the free product $F_{n+1} * t^{\mathbb{Z}}$ induces a map $\phi$ of the homology groups of the kernels $K_{0}(k, d)$ and $K(k, d)$, which is also equivariant for the action of the group ring $\mathbb{Z}[\mathbb{Z} / d \mathbb{Z}]$. Thus we get maps of $R_{d}$ modules $\phi^{n i}: V_{0}^{n i} \simeq R_{d}^{n} \rightarrow V^{n i} \simeq R_{d}^{n+1}$. Since, as $\mathbb{Q}$-vector spaces, the image of $\phi: V_{0} \rightarrow V$ has codimension $\geq$ $(n+1) d-n d=d$, it follows that (at the level of "non-invariants") the image of $V_{0}^{n i}$ in $V^{n i}$ has codimension $\geq d-1$.

6.2. Image of the Gassner Module. The pure braid group $P_{n+1}$ acts on $F_{n+1}$, and acts trivially on the abelian quotient $\mathbb{Z} / d \mathbb{Z}$. We take the trivial action of $P_{n+1}$ on $t^{\mathbb{Z}}$ and get an action of $P_{n+1}$ on $F_{n+1} * t^{\mathbb{Z}}$. Hence $P_{n+1}$ acts on $K_{0}(k, d)$ and on $K(k, d)$. The map $K_{0}(k, d) \rightarrow K(k, d)$ is equivariant for this action as well, and the action of $P_{n+1}$ on the modules $K_{0}(k, d)^{a b}$ and $K(k, d)^{a b}$ is equivariant for the action of the product group $\mathbb{Z} / d \mathbb{Z} \times P_{n+1}$.

We had the exact sequence

$$
1 \rightarrow K \rightarrow F_{n+1} * F_{n+1}^{a b} \rightarrow F_{n+1}^{a b} \rightarrow 1,
$$

and realised $K^{a b}$ as a module over the group algebra $R=\mathbb{Z}\left[F_{n+1}^{a b}\right]$. By replacing $R$ by the larger ring $R^{\prime}=R\left[\frac{1}{\left(1-X_{1}\right) \cdots\left(1-X_{n+1}\right)}\right]$, we found the direct sum decomposition

$$
K^{a b} \otimes_{R} R^{\prime} \simeq W \otimes R^{\prime} \oplus R^{\prime} v_{n+1},
$$

of $R^{\prime}$ modules (but not of $P_{n+1}$ modules). In this decomposition, $W$ was the reduced Gassner representation $g_{n}(X): P_{n+1} \rightarrow G L_{n}(R)$. We now get a map from $F_{n+1} * F_{n+1}^{a b}$ onto $F_{n+1} * t^{\mathbb{Z}}$ given by $x_{i} \mapsto x_{i} \in F_{n+1}$ and $X_{i} \mapsto t^{k_{i}}$. This induces a map from the abelianised kernels $K^{a b}$ into $K(k, d)^{a b}$, with the image of $K^{a b}$ being precisely the specialisation $X_{i} \mapsto t^{k_{i}}$; in other words, the image of $K^{a b}$ is the Gassner representation evaluated at $\left(t^{k_{1}}, \cdots, t^{k_{n+1}}\right)$. It follows that if $v$ is the invariant vector in $K^{a b}$ then

$$
v=\left(1-X_{1}\right) \varepsilon_{1}+\cdots+\left(1-X_{1} X_{2} \cdots X_{n}\right) \varepsilon_{n}+\left(1-X_{1} \cdots X_{n+1}\right) v_{n+1} .
$$


Then its image in $K(k, d)^{a b}$ is also invariant; moreover, if $t_{1} \cdots t_{n+1}=1$ then the image of $v$ lies in the image of $W$, namely it is the image of the element $\sum_{i=1}^{n}\left(1-t_{1} \cdots t_{i}\right) \varepsilon_{i}$.

The polynomials $1-q^{k_{i}}$ and $1+q+\cdots q^{d-1}=\left(1-q^{d}\right) /(1-q)$ are coprime since $k_{i}$ and $d$ are coprime. Therefore, $1-q^{k_{i}}$ is invertible in the ring $R_{d}=\mathbb{Q}[q] /\left(1+q+\cdots q^{d-1}\right)$, and therefore, the map $R \mapsto$ $\mathbb{Q}[q] /\left(q^{d}-1\right)$ induces a ring homomorphism $R^{\prime} \rightarrow R_{d}$ (recall that $R=$ $\mathbb{Z}\left[X_{1}^{ \pm 1}, \cdots, X_{n+1}^{ \pm 1}\right]$ and that $R^{\prime}$ is obtained from $R$ by inverting all the elements $\left.1-X_{i}\right)$. Hence $V^{n i}=H_{1}\left(K(k, d)^{a b}, \mathbb{Q}\right) /\left(1+q+\cdots q^{d-1}\right)$ is naturally a module over $R^{\prime}$. Therefore, $V^{n i}$ is the (full) Gassner representation evaluated at $\left(q^{k_{1}}, \cdots, q^{k_{n+1}}\right)$. The module $V^{n i}$ contains image of the sub-module $W \otimes R^{\prime}$, under the map $R^{\prime} \mapsto R_{d}$. However, $W$ is spanned by the "commutator" elements

$$
\varepsilon_{i}=\frac{1}{\left(1-X_{i}\right)\left(1-X_{i+1}\right)}\left[x_{i}, x_{i+1}\right]
$$

Denote by $\varepsilon_{i}^{\prime}$ the image of $\varepsilon_{i}$ in $V_{0}$. Therefore, $\varepsilon_{i}^{\prime}$ lie in the image of the abelianised kernel $K_{0}(k, d)^{a b} \otimes_{R} R^{\prime}$ (the commutator $\left[x_{i}, x_{i+1}\right]$ certainly lies in the image of $K_{0}(k, d)$; but the inverted elements of $R^{\prime}$ map into elements which have inverses in $R_{d}$ and hence $\varepsilon_{i}^{\prime}$ lies in the image tensored with $\left.R^{\prime}\right)$. Consequently, the image of $V_{0}^{n i}=K_{0}(k, d) \otimes \mathbb{Q}$ has codimension $\leq d-1$ :

$$
V^{n i} /\left(\operatorname{image}\left(W \otimes R^{\prime}\right)=R v /\left(1+q+\cdots+q^{d-1}\right),\right.
$$

and the latter space has dimension $\leq d-1$.

The conclusion of the preceding subsection 6.1 now implies that the module $V_{0}^{n i}$ maps injectively into $V^{n i}$ and that the image is precisely the image of $W \otimes R^{\prime}$, the reduced Gassner representation evaluated at $\left(q^{k_{1}}, \cdots, q^{k_{n+1}}\right)$. We have proved the following

Theorem 24. The representation of $P_{n+1}$ on the homology group

$$
V_{0}^{n i}=\left(K_{0}(d, k)^{a b} \otimes \mathbb{Q}\right) /\left(1+q+\cdots q^{d-1}\right),
$$

is isomorphic to the reduced Gassner representation specialised at $X_{i} \mapsto$ $q^{k_{i}}$, and as a module over $R_{d}$ it is the free module $R_{d}^{n}$ (where $R_{d}$ is the quotient ring $\left.\mathbb{Q}[q] /\left(1+q+\cdots+q^{d-1}\right)\right)$. We have therefore, the decomposition

$$
V_{0}=\bigoplus_{e \mid d, e \geq 2} g_{n}(k, e)
$$

of the (non-invariant part of the) homology of $K_{0}(k, d)$ as a sum of the reduced Gassner representations $g_{n}(k, e)$. 
Denote by $Q_{0}(k, d)$ the quotient of the free group $K_{0}(k, d)$ by the smallest subgroup $N$ normalised by $F_{n+1}$ and containing the "unipotent" elements $x_{1}^{d}, x_{2}^{d}, \cdots, x_{n+1}^{d}$ and the element $\left(x_{1} x_{2} \cdots x_{n+1}\right)^{d / r}$, where $r$ is the g.c.d. of the sum $k_{1}+k_{2}+\cdots+k_{n+1}$ and the number $d$ (If we view $F$ as the fundamental group of the punctures Riemann surface, then $F$ is a subgroup of $S L_{2}(\mathbb{R})$ and the loops $x_{i}$ around the punctures are unipotent elements in $S L_{2}(\mathbb{R})$; this is the reason we have called the $x_{i}$ unipotent elements. We do not use the fact that $F$ may be viewed as a subgroup of $\left.S L_{2}(\mathbb{R})\right)$. The quotient map $K_{0}(k, d) \rightarrow Q_{0}(k, d)$ induces a corresponding map on the $\mathbb{Q}$-homology:

$$
\left.\phi: V_{0}=H_{1}\left(K_{0}(k, d), \mathbb{Q}\right) \rightarrow X_{0}=H_{1}\left(Q_{0}(k, d)\right), \mathbb{Q}\right) .
$$

Since the kernel $N$ to the quotient map is, by assumption, normalised by $F_{n+1}$, it follows that the foregoing map $\phi$ on homology is equivariant for the action of $\mathbb{Z} / d \mathbb{Z}$; therefore, $\phi$ is a map of $\mathbb{Q}[q] /\left(q^{d}-1\right)$ modules. Correspondingly, we get a map

$$
\phi: V_{0}^{n i} \rightarrow X_{0}^{n i}=H_{1}\left(Q_{0}(k, d), \mathbb{Q}\right) /\left(1+q+\cdots+q^{d-1}\right) .
$$

The vector space $X_{0}$ does not have any invariants for the group $\mathbb{Z} / d \mathbb{Z}$, because invariants in the quotients $X_{0}$ are in the image of invariants in $V_{0}$ ( $G$ is a finite group and the modules are $\mathbb{Q}$-vector spaces). Secondly, by equation 11, the only invariants in $V_{0}=H_{1}\left(K_{0}(k, d), \mathbb{Q}\right)$ are the span of the "unipotent" classes $\left[x_{1}^{d}\right], \cdots,\left[x_{n+1}^{d}\right]$ which lie in the kernel of the map $K_{0}(k, d)^{a b} \rightarrow Q_{0}(k, d)^{a b}$. Therefore, $X_{0}^{n i}=X_{0}$.

The action of the group $P_{n+1}$ on the free group $F_{n+1}$ is such that each generator $x_{i}$ of $F_{n+1}$ goes into a conjugate of itself (see subsection (3.2)). Moreover, the product element $x_{1} \cdots x_{n+1}$ is invariant under all of $B_{n+1}$. Hence the normal subgroup $N$ is stable under the action of the pure braid group $P_{n+1}$, and therefore, the homology group $H_{1}\left(Q_{0}(k, d), \mathbb{Q}\right)$ is a $P_{n+1}$ module and the map $\phi$ is equivariant for the action of $P_{n+1}$ as well. We have the following corollary of Theorem 24.

Corollary 3. The representation of $P_{n+1}$ on the quotient $X_{0}$ is a direct sum

$$
X_{0} \simeq \bigoplus_{e \mid d, e \geq 2} \bar{g}_{n}(k, d)
$$

of the quotients $\bar{g}_{n}(k, d)$ of the reduced Gassner representations by the ( possibly one dimensional) space of invariants

Proof. Since $X_{0}=X_{0}^{n i}$ it follows that the elements $x_{i}^{d} \in V_{0}$ map to zero in $X_{0}$. Hence the quotient $X_{0}$ is $V_{0}$ modulo the $\mathbb{Z}[q]$ - module generated 
by $g=\left(x_{1} x_{2} \cdots x_{n+1}\right)^{d / r}$ : the other elements $\left[x_{i}^{d}\right]$ in $V_{0}$ map to zero.

We now deal with the element $\left(x_{1} x_{2} \cdots x_{n+1}\right)^{d / r}$. Recall that $r$ is the g.c.d. of the integers $d$ and $\sum_{i=1}^{n+1} k_{i}$. Then, the element $g_{\infty}=$ $\left(x_{1} x_{2} \cdots x_{n+1}\right)^{d / r}$ viewed as an element of $F_{n+1} / K_{n+1}(d)^{(1)}$ (written multiplicatively) lies in $K_{n+1}(d)^{a b}$. Put $t_{i}=q^{k_{i}}$ and $\pi=t_{1} t_{2} \cdots t_{n+1}$. In $K_{n+1}(k, d)^{a b}$ the element $g_{\infty}$ of the abelian group (written additively) is of the form

$$
w=\left(x_{1} x_{2} \cdots x_{n+1}\right)^{d / r}=v\left(1+\pi+\cdots \pi^{d / r-1}\right)=\lambda v .
$$

In this formula, $v$ is the invariant element in $K^{a b}$ encountered before:

$$
v=\left(1-t_{1}\right) \varepsilon_{1}+\cdots+\left(1-t_{1} t_{2} \cdots t_{n+1}\right) \varepsilon_{n} .
$$

We need only check that this element $g_{\infty}=\lambda v$ goes to zero in the $e$-th component of the decomposition $H_{1}\left(K_{0}(k, d), \mathbb{Q}\right) \simeq \oplus g_{n}(k, e)$, exactly when $g_{n}(k, e)$ has an invariant vector.

If $g_{n}(k, e)$ has an invariant vector, then the element $\pi=t_{1} t_{2} \cdots t_{n+1}$ of $\mathbb{Z}[\mathbb{Z} / d \mathbb{Z}]$ maps to 1 in the quotient $\mathbb{Z} / e \mathbb{Z}$ of the group $G=\mathbb{Z} / d \mathbb{Z}$. Hence the projection to the $e$-th factor of $w$ is a non-zero scalar multiple of the projection of $v$ since $\lambda=d / r \neq 0$ at the $e$-th place.

If $g(k, e)$ does not have an invariant vector, then $\pi=t_{1} t_{2} \cdots t_{n+1}$ is not 1 in $\mathbb{Z} / e \mathbb{Z}$; hence $\lambda=\frac{1-\pi^{e}}{1-\pi}=0$ and our element $g_{\infty}$ goes to zero. We have therefore verified that corresponding to the decomposition

$$
V_{0}=H_{1}\left(K_{0}(k, d), \mathbb{Q}\right) /\left(1+q+\cdots+q^{d-1}\right) \simeq \bigoplus_{e \mid d e \geq 2} g_{n}(k, e)
$$

of representations of the pure braid group $P_{n+1}$, the quotient $X_{0}=$ $V_{0}^{n i} / \mathbb{Q}[G] g_{\infty}$ has a corresponding decomposition

$$
X_{0}=H_{1}\left(Q_{0}(k, d), \mathbb{Q}\right) \simeq \bigoplus_{e \mid d} \overline{g_{n}(k, e)} .
$$

as representations of the pure braid group $P_{n+1}$.

Corollary 4. If $n \geq 2 d$, then the image of the representation of $P_{n+1}$ on the space $W_{0}$ is an arithmetic group.

Proof. For $e \geq 2$ dividing $d$, denote by $G_{e}$ the unitary group of the skew hermitian form $\bar{h}$ on the space $\bar{g}_{n}(k, e)$. We have seen from the preceding corollary that if $\Gamma$ is the image of the action of $P_{n+1}$ on $X_{0}$ then $\Gamma$ is contained in the product $\prod_{e \mid d} G_{e}\left(O_{e}\right)$. Suppose first that 
$e \geq 3$. Then by Theorem 16 (since $n \geq 2 d \geq 2 e$ ), the image of $\Gamma$ in $G_{e}\left(O_{e}\right)$ has finite index. If $e=2$ then the method of Theorem 16] does not apply. However, for $e=2$, is is a well known theorem of [3] that the image of $\Gamma$ in $G_{e}\left(O_{e}\right)=S p_{2 e}(\mathbb{Z})$ has finite index. Therefore, by Lemma 10, $\Gamma$ has finite index in the product $\prod_{e \mid d} G_{e}\left(O_{e}\right)$.

\section{Connection with Monodromy}

7.1. Some Cyclic Coverings of $\mathbb{P}^{1}$. Let $a_{1}, a_{2}, \cdots, a_{n+1}$ be distinct complex numbers; write $S_{a}$ for the complement in $\mathbb{C}$ of these points: $S_{a}=\mathbb{C} \backslash\left\{a_{1}, a_{2}, \cdots, a_{n+1}\right\}$. The fundamental group of $S_{a}$, once a base point is chosen, may be identified with the free group on $F_{n+1}$ generated by small circles $x_{i}$ going around the point $a_{i}$ counterclockwise once (and joined to the preferred base point by an arc which avoids all the points $a_{j}$ and has zero winding number around all the points $a_{j}$ ). The map $S_{a} \rightarrow \mathbb{C}^{*}$ defined by

$$
x \mapsto\left(x-a_{1}\right)^{k_{1}}\left(x-a_{2}\right)^{k_{2}} \cdots\left(x-a_{n+1}\right)^{k_{n+1}}=P_{a}(x),
$$

induces a homomorphism $F_{n+1} \rightarrow q^{\mathbb{Z}}$, which sends each $x_{i}$ to $q^{k_{i}}$. Here, $q$ is a small circle around zero in $\mathbb{C}^{*}$ which runs counterclockwise exactly once.

For future reference, note that the loop around infinity lying in $S_{a}$ represents (the inverse of) the product element $x_{1} x_{2} \cdots x_{n+1}$ and that this element is invariant under the action of the braid group $B_{n+1}$ on the free group $F_{n+1}$.

The affine variety $\mathbb{C}^{*}=\mathbb{G}_{m}$ admits a cyclic covering of order $d$ given by $z \mapsto z^{d}$ from $\mathbb{G}_{m}$ to $\mathbb{G}_{m}$. The covering may be realised as the space $\left\{(x, y) \in \mathbb{C}^{*} \times \mathbb{C}^{*}: y^{d}=x\right\}$ and the covering map is the first projection. Pulling this covering back to $S_{a}$ we get a cyclic covering of $S_{a}$, realised as the space

$X_{a, k}=\left\{(x, y) \in S_{a} \times \mathbb{C}^{*}: y^{d}=\left(x-a_{1}\right)^{k_{1}}\left(x-a_{2}\right)^{k_{2}} \cdots\left(x-a_{n+1}\right)^{k_{n+1}}\right\}$, with the first projection being the covering map from $X_{a}$ onto $S_{a}$. Therefore, under the identification of the fundamental group of $S_{a}$ with $F_{n+1}$, the fundamental group of $X_{a, k}$ is identified with $K_{0}(d, k)$.

As the collection $a$ varies, we get a collection $\mathcal{P}$ of monic polynomials $P_{a}$ of degree $n+1$ which have distinct roots $a_{i}$ occurring with given multiplicities $k_{i}$, and if $\mathcal{Q}$ denotes the variety

$$
(w, x, P) \in \mathbb{C}^{*} \times \mathbb{C} \times \mathcal{P}: w=P(x),
$$


then the projection on to the third coordinate gives a fibration over $\mathcal{P}$ with fibre at $P$ being $S_{a}$ (here $a$ is the collection of roots of $P$ ). We therefore get a monodromy action of the fundamental group of $\mathcal{P}$ on the fundamental group $F_{n+1}$ of the fibre. We have the following basic theorem of E. Artin.

Theorem 25. (Artin) The fundamental group of $\mathcal{P}$ is a subgroup of the Braid Group $B_{n+1}$ and contains the pure braid group $P_{n+1}$. The monodromy action of $P_{n+1}$ on $\pi_{1}\left(S_{a}\right) \simeq F_{n+1}$ is the usual action of $P_{n+1}$ on $F_{n+1}$ defined in section (3.2).

Consequently, the monodromy action on the fibre of the fibration

$$
\left\{(y, x, a) \in \mathbb{C}^{*} \times \mathbb{C} \times \mathcal{C}: y^{d}=\prod\left(x-a_{i}\right)^{k_{i}}\right.
$$

over $\mathcal{C}$ is the usual action of $P_{n+1}$ on the subgroup $K_{n+1}(d) \simeq \pi_{1}\left(X_{a, k}\right)$; therefore, $P_{n+1}$ acts on the first homology $K_{n+1}(k, d)^{a b}$ of $X_{a, k}$, and this gives the monodromy action. Therefore, the first part of Proposition 3 follows from (the Hurewicz Theorem and) Theorem 24.

7.2. The Compactification of $X_{a, k}$. Denote by $X_{a, k}^{*}$ the compactification of the affine $X_{a, k} ; X_{a, k}$ is a compact Riemann surface with finitely many punctures; hence $X_{a, k}^{*}$ is a smooth projective curve obtained by filing in these punctures.

Now the covering map $X_{a, k} \rightarrow S_{a}$ is such that these punctures lie over the points $a_{i}$ or else over the point at infinity of $S_{a}$. If a puncture lies over some $a_{i}$, then the image of a small loop around the puncture in $F_{n+1}$ is $x_{i}^{d}$ (since $k_{i}$ is coprime to $d$ ); if the puncture lies above infinity, then the image of a small loop around the puncture in $F_{n+1}$ is a power of $x_{1} x_{2} \cdots x_{n+1}$ the loop around infinity; therefore, such an element is invariant under the action of the braid group.

The mapping of $\pi_{1}\left(X_{a, k}\right) \rightarrow \pi_{1}\left(X_{a, k}^{*}\right)$ is such that these loops around the punctures generate the kernel, by the van Kampen theorem; consequently, the elements $x_{1}^{d}, \cdots, x_{n+1}^{d}$ map to zero in $H_{1}\left(X_{a, k}^{*}, \mathbb{Z}\right)$ and the element $\left(x_{1} x_{2} \cdots x_{n+1}\right)^{d / r}$ maps to 0 . Therefore, the fundamental group of $X_{a, k}^{*}$ may be identified with the quotient $Q_{0}(k, d)$ of Corollary 3. Therefore, the second part of Proposition 3 follows from Corollary 3 .

The arithmeticity of the monodromy (Theorem 1) now follows from Corollary 4 since the homology of $X_{a, k}^{*}$ is the homology of $Q_{0}(k, d)$.

Acknowledgements: I am very grateful to Madhav Nori for the crucial remark that an earlier proof for the arithmeticity of the image of 
the Burau representation would also go through for the Gassner representation).

The support of the JC Bose fellowship for the period 2008-2013 is gratefully acknowledged.

\section{REFERENCES}

[1] M. Abdulrahim, Complex specialisations of the reduced Gassner representation of the pure braid group, Proc. Amer. Math.Soc, 125 (1997), no. 6, $1617-1624$.

[2] M. Abdurahim, A faithfulness criterion for the Gassner representation of the pure braid group, Proc. Amer. Math. Soc 125, (1997), 1249-1257.

[3] N. A'Campo, Tresses, monodromie et groupes symplectique, Comment. Math. Helvetici, 54 (1987), 318-327.

[4] H.Bass, J.Milnor and J-P. Serre, Solution of the Congruence subgroup problem for $S L_{n}(n \geq 3)$ and $S p_{2 n}(n \geq 2)$, Publ. IHES, 33, (1967), 59-137.

[5] F.Beukers and G. Heckman, Monodromy for the hypergeometric function $n F_{n-1}$, Invent. Math. 95 (1989), 325-354.

[6] J.Birman, Braids, links and mapping class groups, 82, Annals of Math. Studies, Princeton University Press, 1974.

[7] A.Borel and J.Tits, Groupes reductifs, Publ. IHES.27, (1965), 55-150.

[8] P.Deligne and G.D. Mostow, Monodromy of hypergeometric functions and non-lattice integral monodromy, Publ.Math. IHES 63, (1986), 5-89.

[9] P.Griffiths and W.Schmid, Recent deveopements in Hodge theory: a discussion of techniques and results, Discrete subgroups of Lie groups and applications to moduli (Internat. Colloq, Bombay 1973), pp 31-127 Oxford University Press, Bombay 1975.

[10] F.Grunewald and A.Lubotzky, Linear representations of the automorphism group of a free group, GAFA 18 (2009), no.5 1564-1608.

[11] M.Kapovich and J.Millson, Quantization of bending deformations of polygons in $E^{3}$, hypergeometric integrals and the Gassner representation, Canad.Math.Bull. 44 (2001), no. 4, 36-60.

[12] D.D.Long, On linear representations of braid groups, Transactions of the A.M.S. Vol 311, No.2, (1989), 535-560. 
[13] E. Looijenga, Uniformization by Lauricella functions, an overview of the theory of Deligne-Mostow, 207-244, Progr.Math.260 Birkhauser, Basel (2007).

[14] E. Looijenga, Prym Representations of the Mapping Class Groups, Geom. Dedicata 64 (1997), no.1, 60-83.

[15] G.A.Margulis, Discrete subgroups of semi-simple Lie groups, Ergebnisse der Mathematik und ihrer Grenzgebiete 3.Folge. Band 17, Springer-Verlag (1991).

[16] C. Mcmullen, Braid groups and Hodge theory, Math.Annalen, Online First, 27th March, (2012). http:// dx.doi.org/10.1007/s00208-012-0004-2 .

[17] G.D. Mostow, Generalised Picard lattices arising from half-integral conditions, Publ.Math. IHES, 63 (1986), 91-106.

[18] M.V.Nori, A nonarithmetic monodromy group, C.R.Acad.Aci, Paris, Ser I, math.302 (1986), no. 2, 71-72.

[19] M.S. Raghunathan, A note on generators for arithmetic subgroups of algebraic groups, Pacific Journal of Mathematics, 152 (1991), 365-373.

[20] P. Sarnak, Notes on thin groups, MSRI hot topics workshop, Feb 2012).

[21] C.Squier, The Burau representation is unitary, Proc. Amer. Math. Soc. 90 (1984), 199-202.

[22] J. Tits, Syst'eme's generateurs de groupes de congruence, C.R.Acad. Sci. Paris, Serie A 283 (1976), 693.

[23] L. Vaserstein, The structure of classical arithmetic groups of rank greater than 1 (English Translation), Math. USSR, Sbornik 20 (1973), 465-492.

[24] T.N.Venkataramana, On systems of generators for arithmetic subgroups of higher rank groups, Pacific Journal of Math. 166 no.1 (1994), 193-212.

[25] T.N.Venkataramana, Arithmeticity of the Burau representation at roots of unity, (to appear in Annals of Mathematics).

T.N.Venkataramana, School of Mathematics, TiFR, Homi Bhabha Road, Colaba, Mumbai 400005, India

E-mail address: venky@math.tifr.res.in 